EPJ Web of Conferences 7, 01005 (2010)

DOI:10.1051/epjconf/20100701005

(C) Owned by the authors, published by EDP Sciences, 2010

\title{
Condensates and correlations in nuclear matter
}

Gerd Röpke

University of Rostock, Germany

\begin{abstract}
Nuclei in dense matter are influenced by the medium. Solving an A-particle Schroedinger equation including the effects of self-energy and Pauli blocking, a quasiparticle description is introduced. Deriving thermodynamic properties, this approach contains the NSE at low densities as well as mean-field approaches at high densities. Consequences for the symmetry energy, the phase transition, the determination of thermodynamic parameters from cluster yields and astrophysical applications are discussed.
\end{abstract}

This is an Open Access article distributed under the terms of the Creative Commons Attribution-Noncommercial License 3.0, which permits unrestricted use, distribution, and reproduction in any noncommercial medium, provided the original work is properly cited. 
HISS: Dense Matter in HIC and Astrophysics, Dubna, 18./21. 7. 08

\title{
Condensates and Correlations in Nuclear Matter
}

\author{
Gerd Röpke \\ Universität Rostock
}

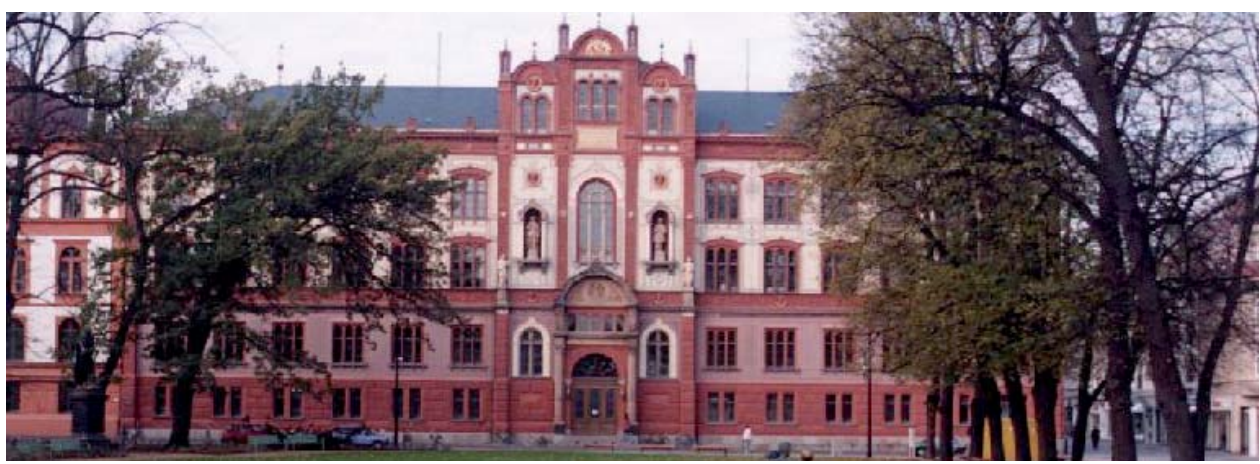

\section{Problems:}

- Warm Dilute Matter: Nuclear matter at subsaturation densities:

Temperature $T \leq 16 \mathrm{MeV}=\mathrm{E}_{\mathrm{s}} / \mathrm{A}$, baryon density $\mathrm{n}_{\mathrm{B}} \leq 0.17 \mathrm{fm}^{-3}=\mathrm{n}_{\mathrm{s}}$.

- Formation of clusters (nuclei in matter):

$A=1,2,3,4$ : deuterons $(d)$, tritons $(t)$, helions $(h)$, alphas $(\alpha)$

- Low-density, low-temperature limit:

Virial expansion, non-interacting nuclides, quantum condensates

- Transition to higher densities:

Medium effects, quasiparticles,

interpolation between Beth-Uhlenbeck and DBHF / RMF

Refs:

Particle clustering and Mott transition in nuclear matter at finite temperatures,

G. Röpke, M. Schmidt, L. Münchow, H. Schulz: NPA 399, 587-602 (1983).

Generalized Beth-Uhlenbeck Approach for Hot Nuclear Matter,

M. Schmidt, G. Röpke, H. Schulz: Annals of Physics 202, 57 - 99 (1990). 


\section{Outline}

- Schrödinger equation with medium corrections:

Self-energy and Pauli blocking

- Composition of the nuclear gas:

Generalized Beth-Uhlenbeck equation

- Quantum condensates:

Pairing and quartetting

- Composition and the EoS of nuclear matter

(astrophysics: supernovae explosions)

- Cluster formation in dilute nuclei

(Hoyle state and THSR wave function)

- Symmetry energy in the low-density region

(heavy ion collisions: cluster abundances)

\section{Low-density EoS and astrophysics}

- H. Shen, H. Toki, K. Oyamatsu, and K. Sumiyoshi, Progr. Theor. Phys.100, 1013 (1998); Nucl. Phys. A637 435 (1998).

- G. Röpke, A. Grigo, K. Sumiyoshi, and Hong Shen, in: Superdense QCD Matter and Compact Stars, Ed. D. Blaschke and A. Sedrakian, NATO Science Series, Springer, Dordrecht (2006), pp. 75 - 91;

Physics of Particles and Nuclei Letters 2, 275 (2005).

- J.M.Lattimer and F. D. Swesty, Nucl. Phys. A 535, 331 (2001).

- C. J. Horowitz and A. Schwenk, Nucl. Phys. A 776, 55 (2006). 


\section{Neutron stars}

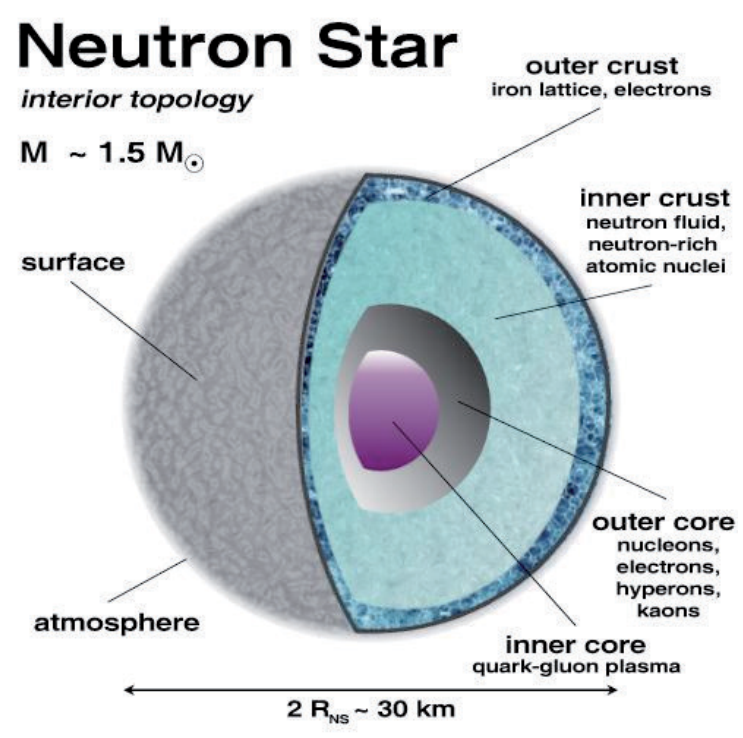

\section{Structure of a Neutron star}

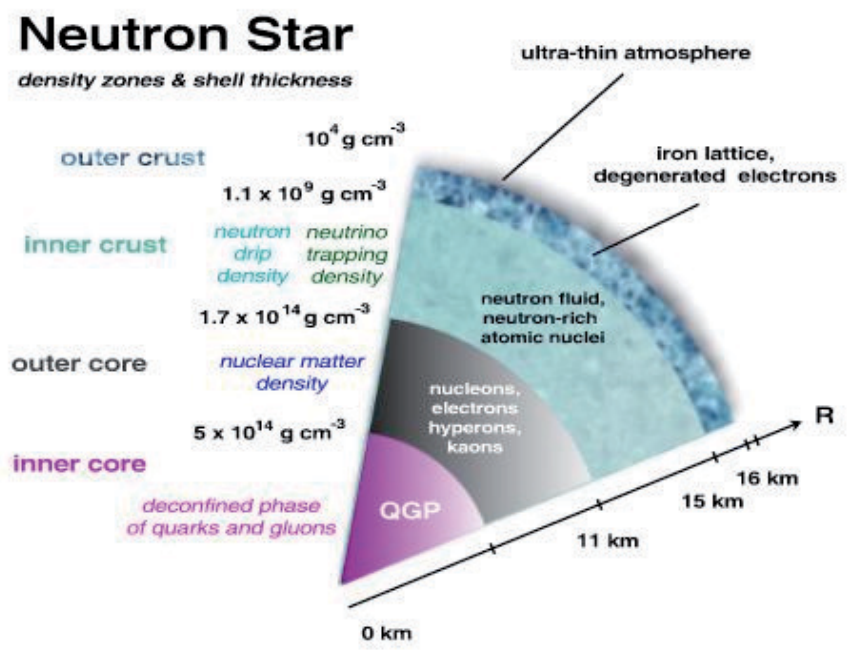




\section{Supernova}

Crab nebula, 1054 China, PSR 0531+21

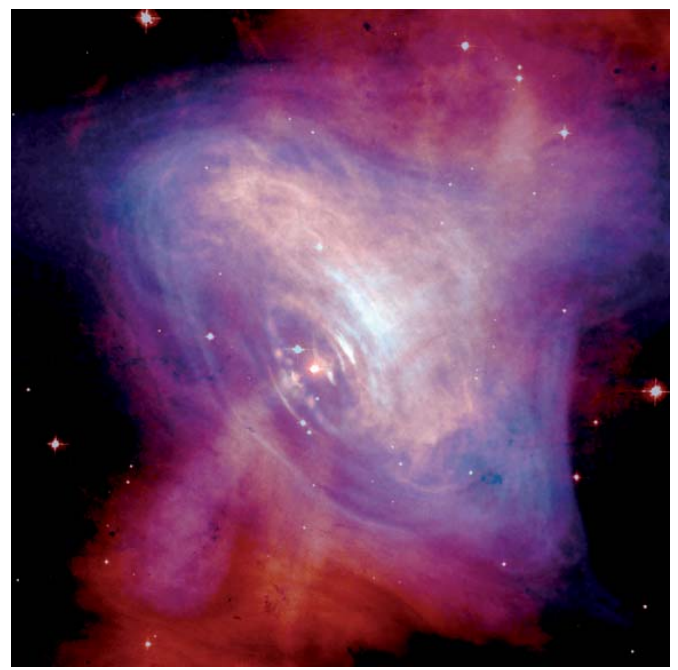

\section{Supernova collapse:}

\section{spherically symmetric simulations}
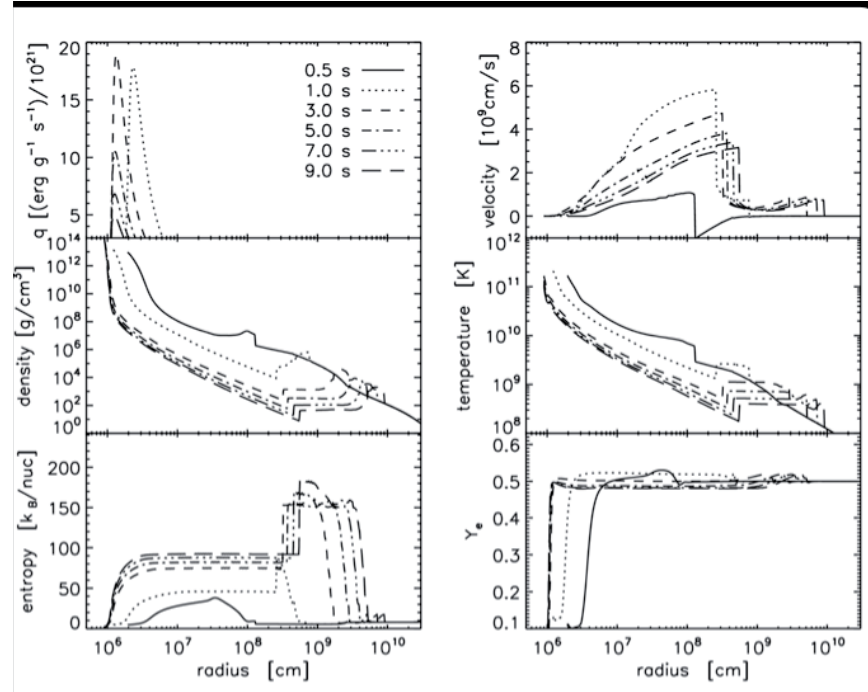

A. Arcones

Neutrino driven winds Talk 25. 2. 08 Ladek 


\section{Core-collapse supernovae}

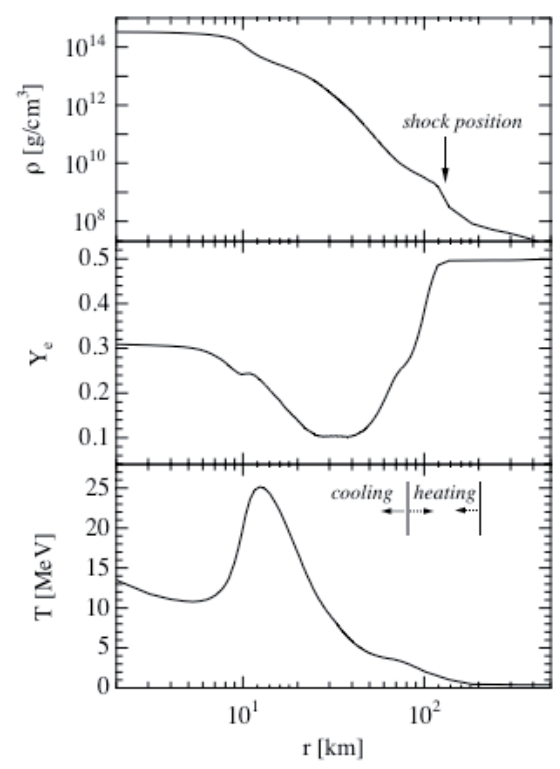

Density.

electron fraction, and

temperature profile

of a 15 solar mass supernova at $150 \mathrm{~ms}$ after core bounce as function of the radius.

Influence of cluster formation on neutrino emission in the cooling region and on neutrino absorption in the heating region?

\section{Parameter range: Explosion}

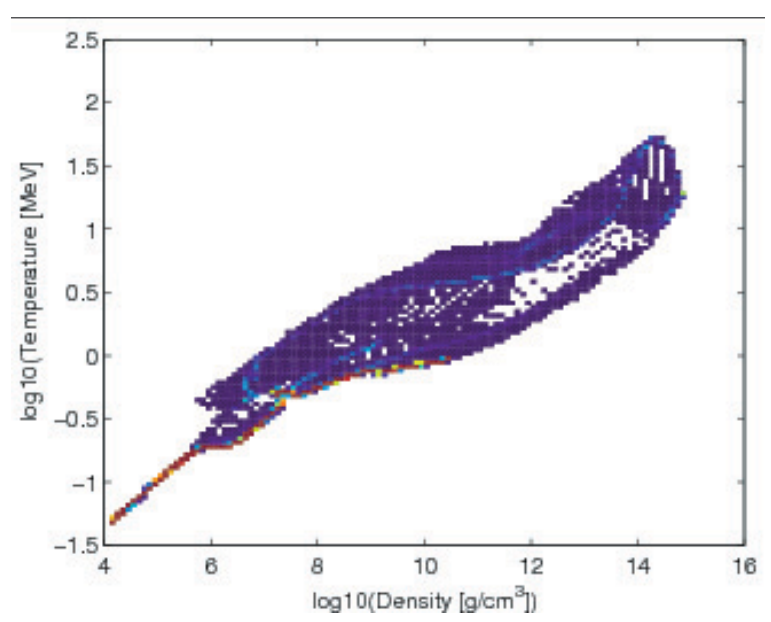

T. Fischer, On the possible fate of massive progenitor stars, Talk 25.2.08 Ladek 


\section{Phase diagram: nuclear matter}

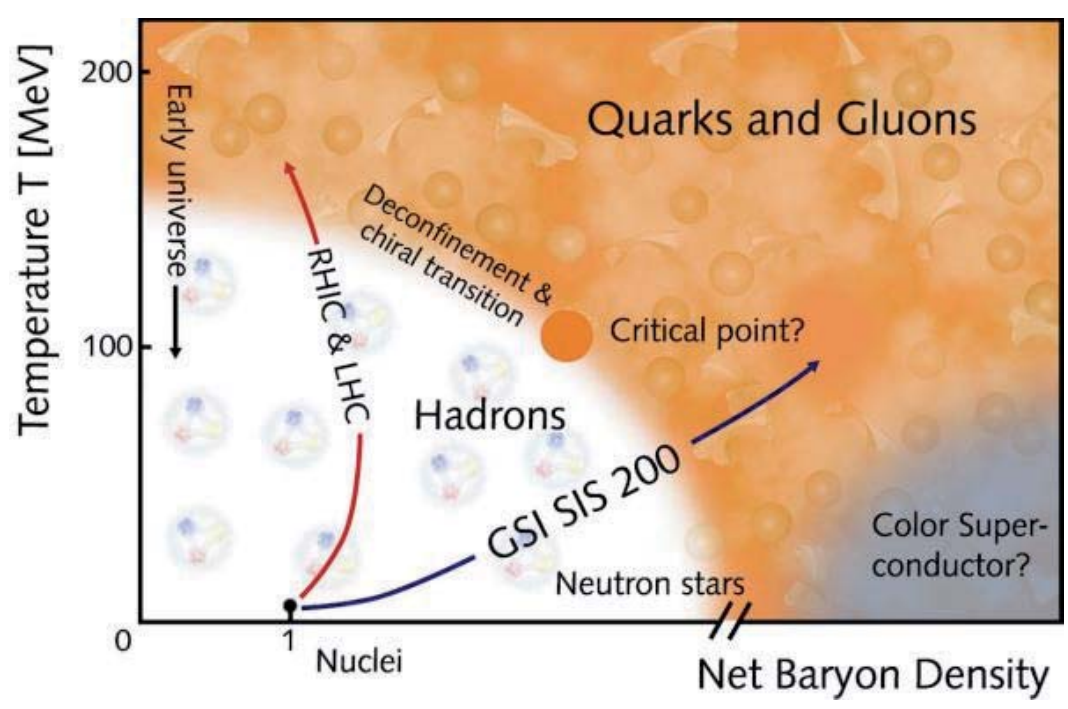

Symmetric nuclear matter: Phase diagram

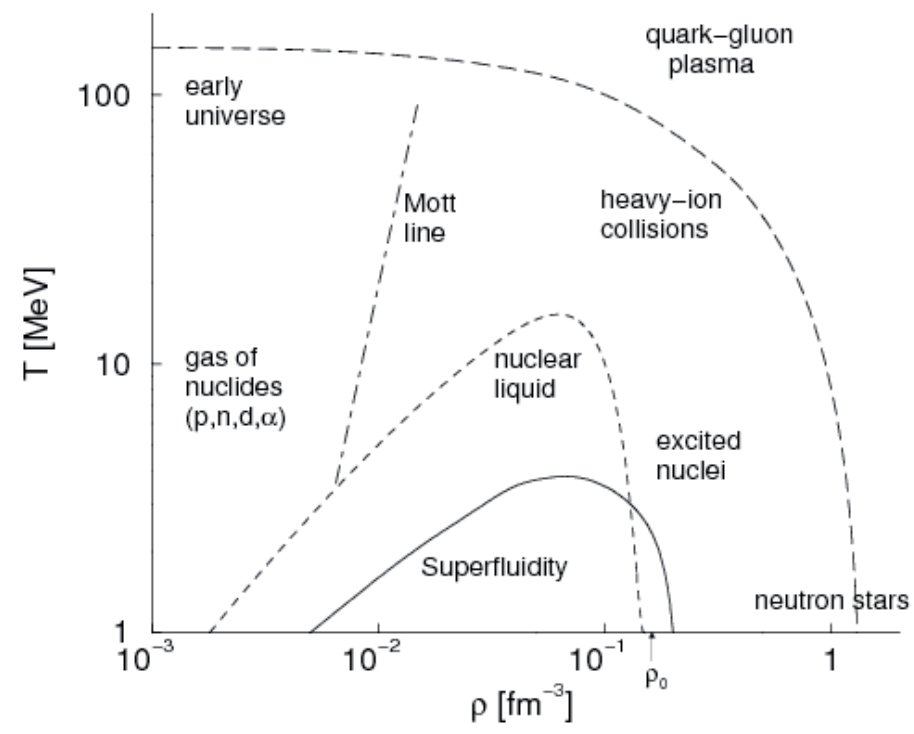




\section{Correlations in low-density matter}

- Ideal fermion gas of protons and neutrons?

- Formation of bound states: nuclei

- Medium modifications: quasiparticle concept

- Relativistic mean field approach, nucleons

- Cluster - mean field approach

\section{Properties of light clusters}

\begin{tabular}{l|r|c|c|c}
\hline & binding energy & mass & spin & rms-radius \\
\hline$n$ & 0 & $939.565 \mathrm{MeV} / c^{2}$ & $1 / 2$ & $0.34 \mathrm{fm}$ \\
$p$ & 0 & $938.783 \mathrm{MeV} / c^{2}$ & $1 / 2$ & $0.87 \mathrm{fm}$ \\
$d$ & $-2.225 \mathrm{MeV}$ & $1876.12 \mathrm{MeV} / c^{2}$ & 1 & $2.17 \mathrm{fm}$ \\
$t$ & $-8.482 \mathrm{MeV}$ & $2809.43 \mathrm{MeV} / c^{2}$ & $1 / 2$ & $1.70 \mathrm{fm}$ \\
$h$ & $-7.718 \mathrm{MeV}$ & $2809.41 \mathrm{MeV} / c^{2}$ & $1 / 2$ & $1.87 \mathrm{fm}$ \\
$\alpha$ & $-28.3 \mathrm{MeV}$ & $3728.40 \mathrm{MeV} / c^{2}$ & 0 & $1.63 \mathrm{fm}$
\end{tabular}




\section{Ideal mixture of reacting nuclides}

$$
\begin{aligned}
& n_{p}\left(T, \mu_{p}, \mu_{n}\right)=\frac{1}{V} \sum_{A, \nu, K} Z_{A} f_{A}\left\{E_{A, \nu K}-Z_{A} \mu_{p}-\left(A-Z_{A}\right) \mu_{n}\right\} \\
& n_{n}\left(T, \mu_{p}, \mu_{n}\right)=\frac{1}{V} \sum_{A, \nu, K}\left(A-Z_{A}\right) f_{A}\left\{E_{A, \nu K}-Z_{A} \mu_{p}-\left(A-Z_{A}\right) \mu_{n}\right\}
\end{aligned}
$$

mass number $A$, charge $Z_{A}$,

$$
f_{A(z)}=\frac{1}{\exp (z / T)-(-1)^{A}}
$$

energy $E_{A, v, K}$,

$v$ internal quantum number,

$\sim K$ center of mass momentum

\section{Composition of nuclear matter}

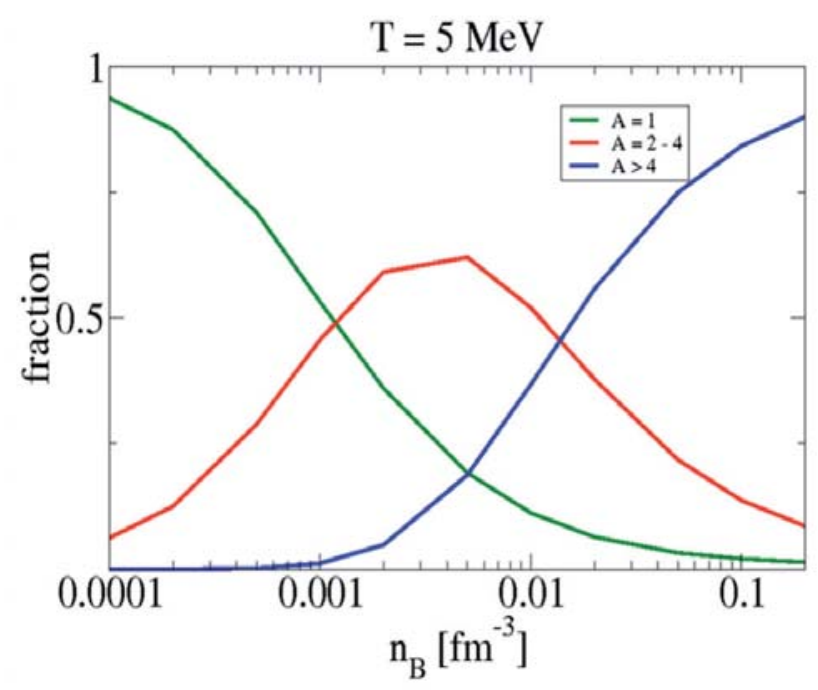




\section{Virial expansion}

- excited nuclei

- resonances

- scattering phase shifts (no double counting)

- virial expansions

- quantum statistical approach

\section{Beth-Uhlenbeck formula}

rigorous results at low density: virial expansion

Beth-Uhlenbeck formula

$$
\begin{aligned}
n(T, \mu) & =\frac{1}{V} \sum_{p} e^{-(E(p)-\mu) / k_{B} T} \\
& +\frac{1}{V} \sum_{n P} e^{-\left(E_{n P}-2 \mu\right) / k_{B} T} \\
& +\frac{1}{V} \sum_{\alpha P} \int_{0}^{\infty} \frac{d E}{2 \pi} e^{-\left(E+P^{2} / 4 m-2 \mu\right) / k_{B} T} \frac{d}{d E} \delta_{\alpha}(E) \\
& +\cdots
\end{aligned}
$$

$\delta_{\alpha}(E)$ : scattering phase shifts, channel $\alpha$ 


\section{Alpha-particle fraction in the low-density limit} symmetric matter, $\mathrm{T}=4 \mathrm{MeV}$

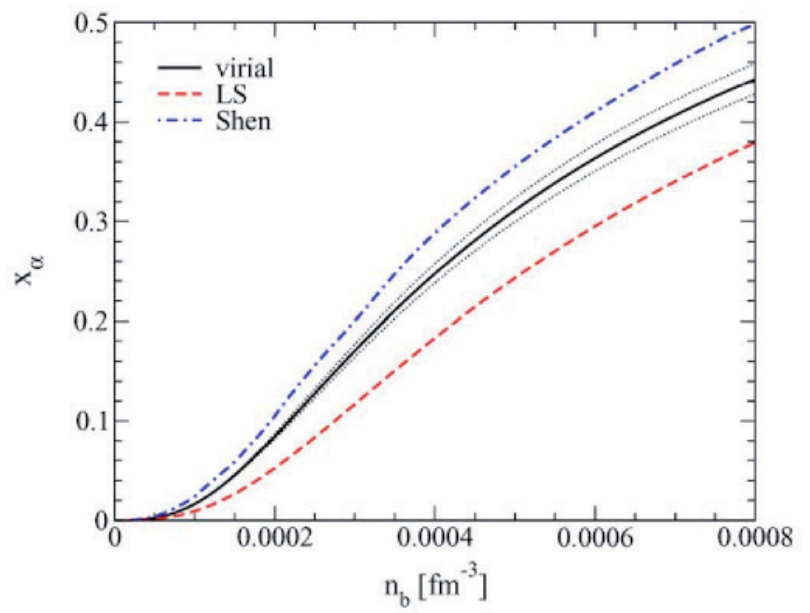

Horowitz \& Schwenk (2006), Lattimer \& Swesty, (2001), Shen et al. (1998))

\section{alpha - fraction at $\mathrm{T}=4 \mathrm{MeV}$}

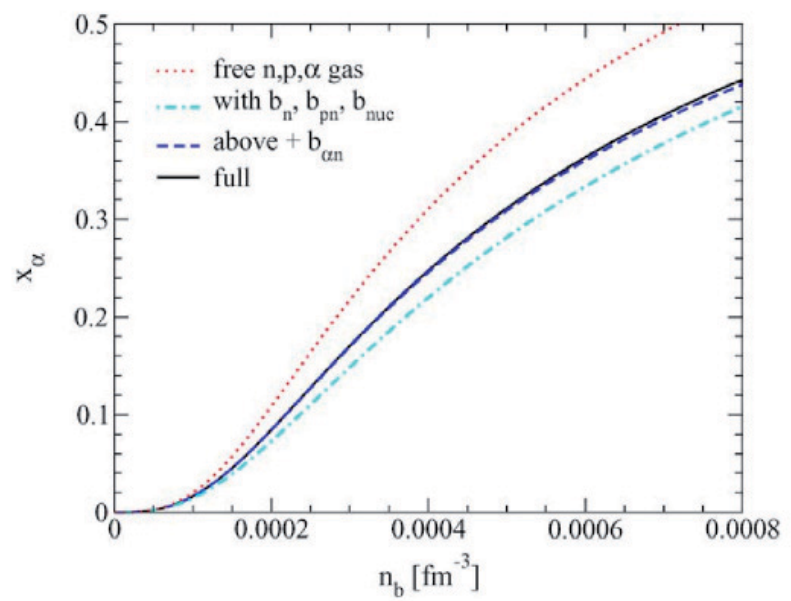

C.J.Horowitz, A.Schwenk, Nucl. Phys. A 776, 55 (2006) 


\section{Nucleon-nucleon interaction}

- general form:

$$
\begin{array}{rlr}
V_{\alpha}\left(p, p^{\prime}\right) & =\sum_{i, j=1}^{N} w_{\alpha i}(p) \lambda_{\alpha i j} w_{\alpha j}\left(p^{\prime}\right) & \text { uncoupled } \\
\text { and } & \\
V_{\alpha}^{L L^{\prime}}\left(p, p^{\prime}\right) & =\sum_{i, j=1}^{N} w_{\alpha i}^{L}(p) \lambda_{\alpha i j} w_{\alpha j}^{L^{\prime}}\left(p^{\prime}\right) & \text { coupled }
\end{array}
$$

$$
\begin{array}{lll}
p, p^{\prime} & \text { in- and outgoing relative momentum } \\
\alpha & \ldots & \text { channel } \\
N \quad \ldots & \text { rank } \\
\lambda_{\alpha i j} & \cdot \text { coupling parameter } \\
L, L^{\prime} & \text { orbital angular momentum }
\end{array}
$$

\section{Separable nucleon-nucleon interaction}

- examples:

- Yamaguchi potential

Y. Yamaguchi, Phys. Rev. 95, 1628 (1954)

rank $=1$, uncoupled, only $S$-waves

- PARIS-potential, PEST $N$

J. Haidenbauer and W. Plessas, Phys. Rev. C 30, 1822 (1984)

consideration of partial waves up to $L=2$

- BONN-potential, BEST $N$

Plessas et al., Few-Body Syst. Suppl. 7, 251 (1994)

consideration of partial waves up to $L=3$ 


\section{Many-particle theory}

- equilibrium correlation functions

e.g. equation of state $n(\beta, \mu)=\frac{1}{\Omega_{0}} \sum_{1}\left\langle a_{1}^{\dagger} a_{1}\right\rangle$

density matrix $\left\langle a_{1}^{\dagger} a_{1}^{\ddagger}\right\rangle=\int \frac{\mathrm{d} \omega}{2 \pi} \mathrm{e}^{-i \omega t} f_{1}(\omega) A\left(1,1^{\prime}, \omega\right)$

- Spectral function

$$
A\left(1,1^{\prime}, \omega\right)=\operatorname{Im}\left[G\left(1,1^{\prime}, \omega+i \eta\right)-G\left(1,1^{\prime}, \omega-i \eta\right)\right]
$$

- Matsubara Green function

$$
\begin{array}{r}
G\left(1,1^{\prime}, i z_{\nu}\right), \quad z_{\nu}=\frac{\pi \nu}{\beta}+\mu, \quad \nu= \pm 1, \pm 3, \cdots \\
1 \equiv\left\{\mathbf{p}_{1}, \sigma_{1}, c_{1}\right\}, \quad f_{1}(\omega)=\frac{1}{\mathrm{e}^{\beta(\omega-\mu)}+1}, \quad \Omega_{0}-\text { volume }
\end{array}
$$

\section{Many-particle theory}

- Dyson equation and self energy (homogeneous system)

$$
G\left(1, i z_{\nu}\right)=\frac{1}{i z_{\nu}-E(1)-\Sigma\left(1, i z_{\nu}\right)}
$$

- Evaluation of $\Sigma\left(1, i z_{\nu}\right)$ :

perturbation expansion, diagram representation

$$
A(1, \omega)=\frac{2 \operatorname{Im} \Sigma(1, \omega+i 0)}{[\omega-E(1)-\operatorname{Re} \Sigma(1, \omega)]^{2}+[\operatorname{Im} \Sigma(1, \omega+i 0)]^{2}}
$$

approximation for approximation for self energy equilibrium correlation functions

alternatively: simulations, path integral methods 


\title{
Different approximations
}

- Expansion for small $\operatorname{Im} \Sigma(1, \omega+i \eta)$

$$
\begin{aligned}
A(1, \omega) \approx & \frac{2 \pi \delta\left(\omega-E^{\text {quasi }}(1)\right)}{1-\left.\frac{\mathrm{d}}{\mathrm{d} z} \operatorname{Re} \Sigma(1, z)\right|_{z=E^{\text {quasi }}-\mu_{1}}} \\
& -2 \operatorname{Im} \Sigma(1, \omega+i \eta) \frac{\mathrm{d}}{\mathrm{d} \omega} \frac{P}{\omega+\mu_{1}-E^{\text {quasi }}(1)}
\end{aligned}
$$

quasiparticle energy $E^{\text {quasi }}(1)=E(1)+\left.\operatorname{Re} \Sigma(1, \omega)\right|_{\omega=E^{\text {quasi }}}$

- chemical picture: bound states $\hat{=}$ new species

summation of ladder diagrams, Bethe-Salpeter equation

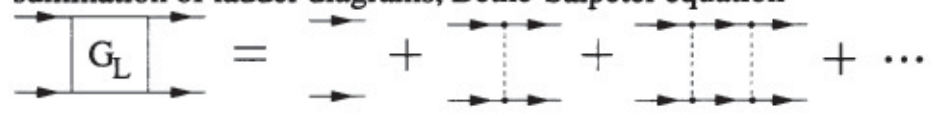

\section{Medium effects}

\author{
Quasiparticle approximation
}




\section{Lagrangian: non-linear sigma}

$$
\begin{aligned}
\mathcal{L}= & \bar{\psi}_{i}\left[i \gamma_{\mu} \partial^{\mu}-m_{i}-g_{\sigma} \sigma-g_{\omega} \gamma_{\mu} \omega^{\mu}-g_{\rho} \gamma_{\mu} \tau_{a} \rho_{a}^{\mu}\right] \psi_{i} \\
& +\frac{1}{2} \partial_{\mu} \sigma \partial^{\mu} \sigma-\frac{1}{2} m_{\sigma}^{2} \sigma^{2}-\frac{1}{3} g_{2} \sigma^{3}-\frac{1}{4} g_{3} \sigma^{4} \\
& -\frac{1}{4} W_{\mu \nu} W^{\mu \nu}+\frac{1}{2} m_{\omega}^{2} \omega_{\mu} \omega^{\mu}+\frac{1}{4} c_{3}\left(\omega_{\mu} \omega^{\mu}\right)^{2} \\
& -\frac{1}{4} R_{\mu \nu}^{a} R^{a \mu \nu}+\frac{1}{2} m_{\rho}^{2} \rho_{\mu}^{a} \rho_{a}^{\mu} .
\end{aligned}
$$

\section{TM1 parameters}

- Shen et al. (1998)

\begin{tabular}{c|r|l}
\hline Parameter & Numerical & value \\
\hline$m_{p}$ & 938.783 & $\mathrm{MeV}$ \\
$m_{n}$ & 939565 & $\mathrm{MeV}$ \\
$m_{\sigma}$ & 511.19777 & $\mathrm{MeV}$ \\
$m_{\omega}$ & 783.0 & $\mathrm{MeV}$ \\
$m_{\rho}$ & 770.0 & $\mathrm{MeV}$ \\
$g_{\sigma}$ & 10.02892 & \\
$g_{\omega}$ & 12.61394 & \\
$g_{\rho}$ & 4.63219 & \\
$g_{2}$ & -7.23247 & $\mathrm{fm}^{-1}$ \\
$g_{3}$ & 0.61833 & \\
$c_{3}$ & 71.30747 &
\end{tabular}




\section{Quasiparticle energies}

- Skyrme

- relativistic mean field (RMF)

$$
\begin{aligned}
e_{i}(k) & =\sqrt{\left(m_{i} c^{2}-S\left(n_{B}, \delta, T\right)\right)^{2}+\hbar^{2} c^{2} k^{2}}+V_{i}\left(n_{B}, \delta, T\right) \\
n_{i} & =\frac{1}{\pi^{2}} \int_{0}^{\infty} d k \frac{k^{2}}{\exp \left[e_{i}(k) / k_{B} T-\mu_{i} / k_{B} T\right]+1}
\end{aligned}
$$

\section{Single particle modifications}

- effective mass

$$
m_{i}^{*}=m_{i}+g_{\sigma} \sigma_{0},
$$

- energy shift

$$
\begin{aligned}
& E_{p}\left(k ; T, \mu_{p}, \mu_{n}\right)=\sqrt{k^{2}+m_{p}^{* 2}}+g_{\omega} \omega_{0}+g_{\rho} \rho_{0}, \\
& E_{n}\left(k ; T, \mu_{p}, \mu_{n}\right)=\sqrt{k^{2}+m_{n}^{* 2}}+g_{\omega} \omega_{0}-g_{\rho} \rho_{0}
\end{aligned}
$$




\section{DD-RMF (Typel, 2007)}

- scalar field

$$
\begin{aligned}
S\left(n_{B}, \delta, T\right)= & n_{B}\left[(4524.13-6.926 T)-14.5157 / 4 \delta^{2}+0.833943 / 16 \delta^{4}-9.00693 / 64 \delta^{6}\right] \\
& +n_{B}^{2}\left[-19190.7-2426.57 / 4 \delta^{2}-317.732 / 16 \delta^{4}-1547.38 / 64 \delta^{6}\right] \\
& +n_{B}^{3}\left[62169.5+2521.29 / 4 \delta^{2}+3470.28 / 16 \delta^{4}\right] \\
& +n_{B}^{4}\left[-91005.1+3984.82 / 4 \delta^{2}-9148.6 / 16 \delta^{4}\right]
\end{aligned}
$$

- vector fields

$$
\begin{aligned}
V_{p}\left(n_{B}, \delta\right)= & V_{n}\left(n_{B},-\delta\right)=n_{B}\left[3462.24+946.705 / 2 \delta-0.334508 / 4 \delta^{2}\right] \\
& +n_{B}^{2}\left[-11312.4-6246.21 / 2 \delta-6353.53 / 4 \delta^{2}-0.099478 / 8 \delta^{3}\right] \\
& +n_{B}^{3}\left[20806.1+18717.6 / 2 \delta+29298 . / 4 \delta^{2}-0.490543 / 8 \delta^{3}\right] \\
& +n_{B}^{4}\left[352.371-24887.2 / 2 \delta-39807.4 / 4 \delta^{2}-0.346218 / 8 \delta^{3}\right] .
\end{aligned}
$$

Quasiparticle approximation for nuclear matter

\section{Equation of state for symmetric matter}

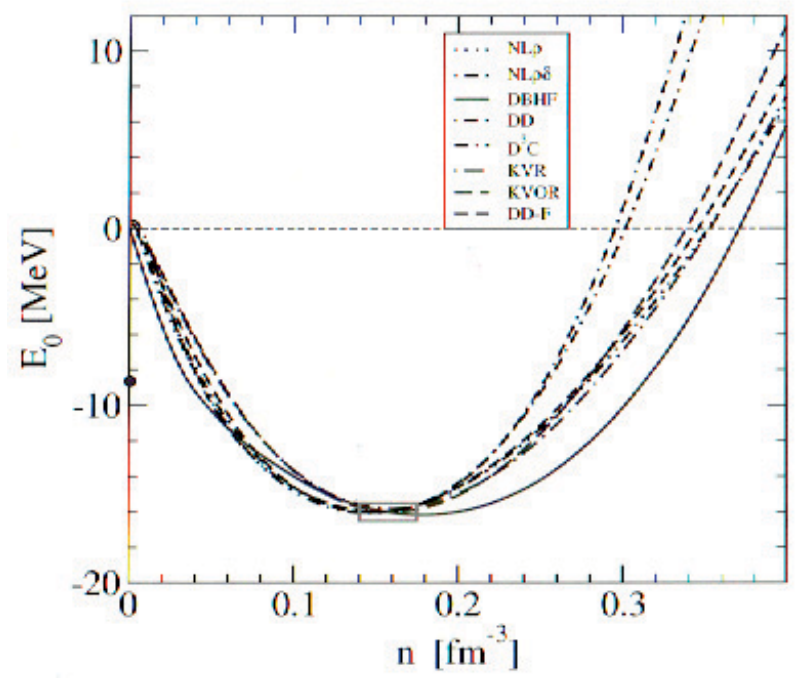




\section{RMF and DBHF}
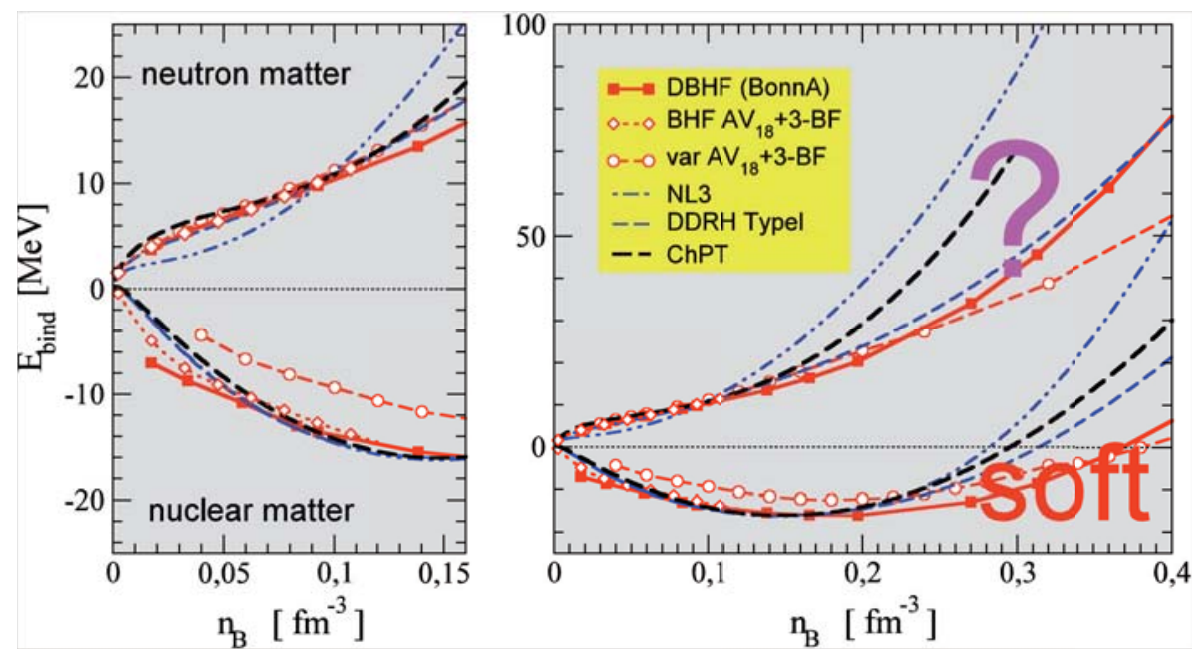

C. Fuchs et al. (2007)

\section{Different approximations}

- Expansion for small $\operatorname{Im} \Sigma(1, \omega+i \eta)$

$$
\begin{aligned}
A(1, \omega) \approx & \frac{2 \pi \delta\left(\omega-E^{\text {quasi }}(1)\right)}{1-\left.\frac{\mathrm{d}}{\mathrm{d} z} \operatorname{Re} \Sigma(1, z)\right|_{z=E^{\text {quasi }}-\mu_{1}}} \\
& -2 \operatorname{Im} \Sigma(1, \omega+i \eta) \frac{\mathrm{d}}{\mathrm{d} \omega} \frac{P}{\omega+\mu_{1}-E^{\text {quasi }}(1)}
\end{aligned}
$$

quasiparticle energy $E^{\text {quasi }}(1)=E(1)+\left.\operatorname{Re} \Sigma(1, \omega)\right|_{\omega=E^{\text {quasi }}}$

- chemical picture: bound states $\hat{=}$ new species

summation of ladder diagrams, Bethe-Salpeter equation

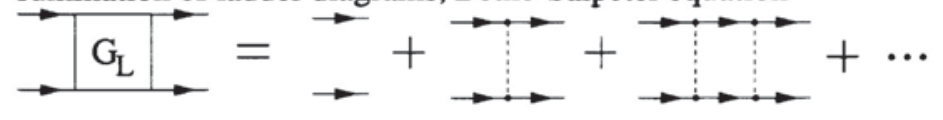




\section{Different approximations}

low density limit:

$$
\begin{aligned}
& G_{2}^{L}\left(12,1^{\prime} 2^{\prime}, i \lambda\right)=\sum_{n \mathbf{P}} \Psi_{n \mathbf{P}}(12) \frac{1}{i \omega_{\lambda}-E_{n \mathbf{P}}} \Psi_{n \mathbf{P}}^{*}(12) \\
& n(\beta, \mu)=\sum_{1} f_{1}\left(E^{q u a s i}(1)\right)+\sum_{2, n \mathbf{P}}^{\text {bound }} g_{12}\left(E_{n \mathbf{P}}\right) \\
& +\sum_{2, n \mathbf{P}} \int_{0}^{\infty} d k \delta_{\mathbf{k}, \mathbf{p} 1-\mathbf{p}_{2}} g_{12}\left(E^{\text {quasi }}(1)+E^{\text {quasi }}(2)\right) 2 \sin ^{2} \delta_{n}(k) \frac{1}{\pi} \frac{\mathrm{d}}{\mathrm{d} k} \delta_{n}(k) \\
& \text { - generalized Beth-Uhlenbeck formula } \\
& \text { correct low density/low temperature limit: } \\
& \text { mixture of free particles and bound clusters }
\end{aligned}
$$

\section{Effective wave equation for the deuteron in matter}

$$
\begin{array}{r}
\left(\frac{p_{1}^{2}}{2 m_{1}}+\frac{p_{2}^{2}}{2 m_{2}}\right) \Psi_{n, P}\left(p_{1}, p_{2}\right)+\sum_{p_{1}{ }^{\prime}, p_{2}{ }^{\prime}}\left(1-f_{p_{1}}-f_{p_{2}}\right) V\left(p_{1}, p_{2} ; p_{1}{ }^{\prime}, p_{2}{ }^{\prime}\right) \Psi_{n, P}\left(p_{1}{ }^{\prime}, p_{2}{ }^{\prime}\right) \\
\text { Pauli-blocking }
\end{array}
$$

Fermi distribution function

$$
f_{p}=\left[e^{\left(p^{2} / 2 m-\mu\right) / k_{B} T}+1\right]^{-1}
$$




\section{Deuterons in nuclear matter}

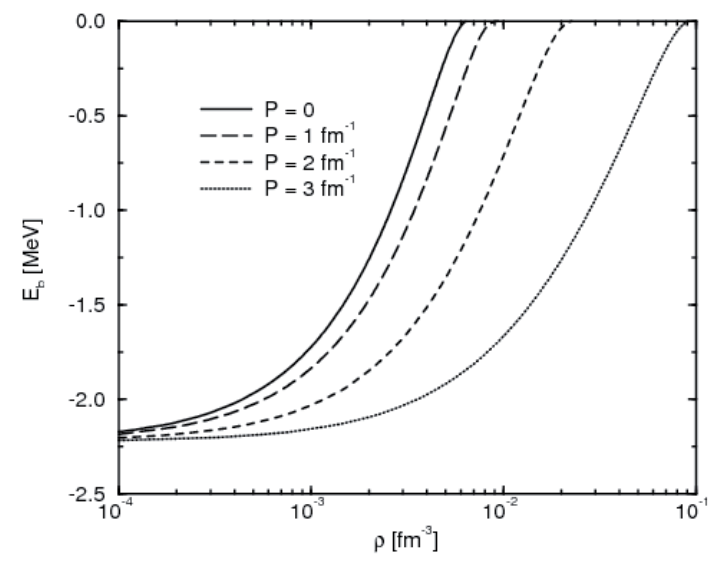

$\mathrm{T}=10 \mathrm{MeV}, \mathrm{P}$ : center of mass momentum

\section{Scattering phase shifts in matter}

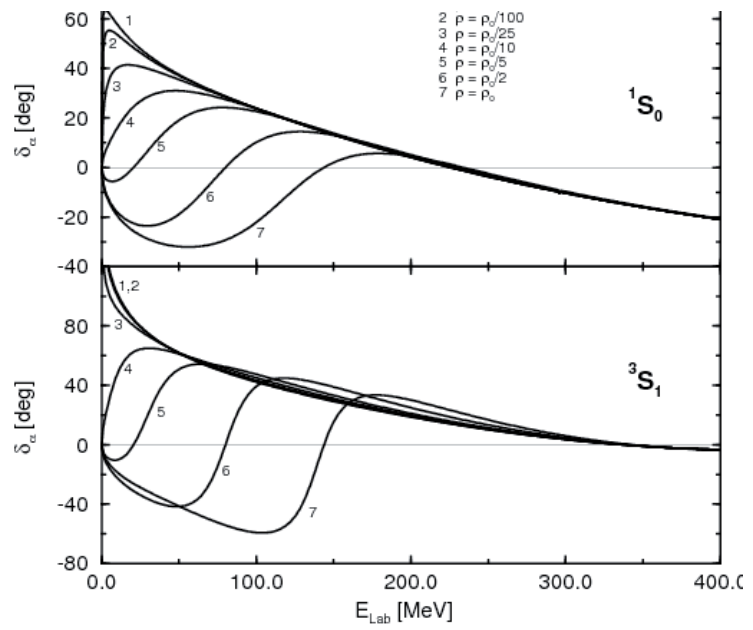




\section{Generalized Beth-Uhlenbeck formula}

- quantum gas

- medium modifications

$$
n(T, \mu)=n_{1}(T, \mu)+n_{2}(T, \mu)
$$

contribution of free quasiparticles

$$
n_{1}(T, \mu)=\frac{1}{V} \sum_{p} \frac{1}{e^{\left(E^{\mathrm{HF}}(p)-\mu\right) / k_{B} T}+1}
$$

$\left(E^{\mathrm{HF}}(p)\right.$ : quasiparticle energy)

contribution of two-particle correlations

$$
n_{2}(T, \mu)=n_{2}^{\text {bound }}(T, \mu)+n_{2}^{\text {scatt }}(T, \mu)
$$

\section{Generalized Beth-Uhlenbeck formula}

$$
\begin{aligned}
n_{2}^{\text {bound }}(T, \mu) & =\frac{1}{V} \sum_{n P} \frac{1}{e^{\left(E_{n P}^{\text {mean field }}-2 \mu\right) / k_{B} T}-1}, \\
n_{2}^{\text {scatt }}(T, \mu) & =\frac{1}{V} \sum_{\alpha P} \int_{0}^{\infty} \frac{d E}{2 \pi} \frac{1}{e^{\left(E_{\alpha P}^{\text {mean field }}+E-2 \mu\right) / k_{B} T}-1} \sin ^{2}\left(\delta_{\alpha}(E)\right) \frac{d}{d E} \delta_{\alpha}(E)
\end{aligned}
$$

Composition: (ionization degree)

correlated part $\frac{n_{2}(T, \mu)}{n(T, \mu)}$

Bose distribution function: Quantum condensates 


\section{Composition of symmetric nuclear matter}

Fraction of

correlated matter

(virial expansion,

Generalized Beth-

Uhlenbeck approach,

contribution

of bound states,

of scattering states,

phase shifts)

H. Stein et al.,

Z. Phys. A351, 259 (1995)

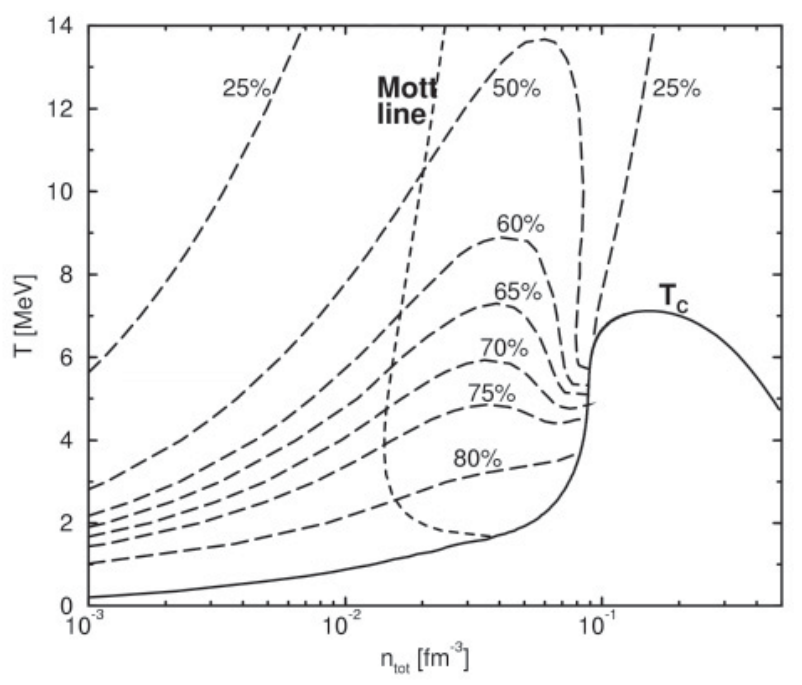

\section{Quantum condensate}

\section{Bose-Einstein- Condensation of deuterons (BEC)}

\section{Bardeen-Cooper} Schrieffer pairing (BCS)

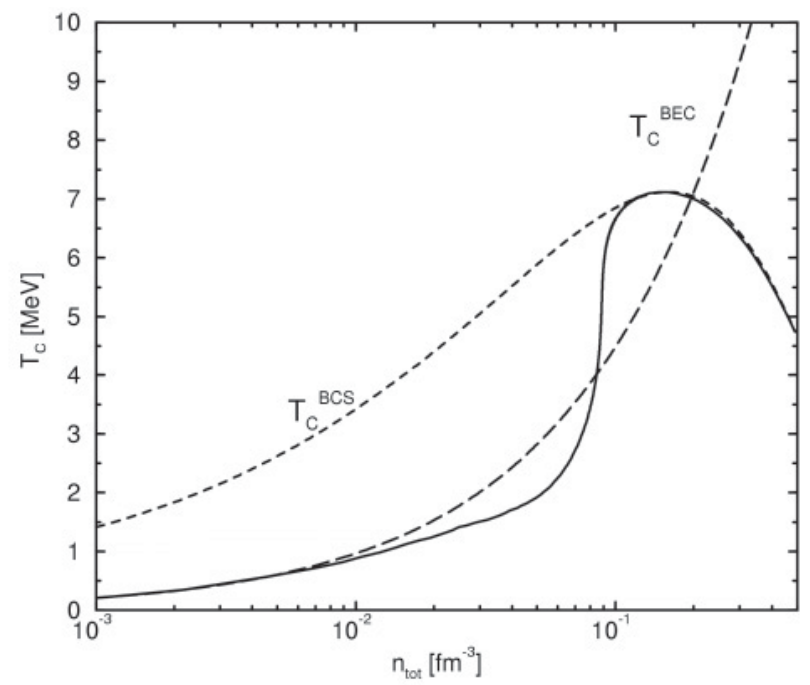




\section{Correlations in the medium}

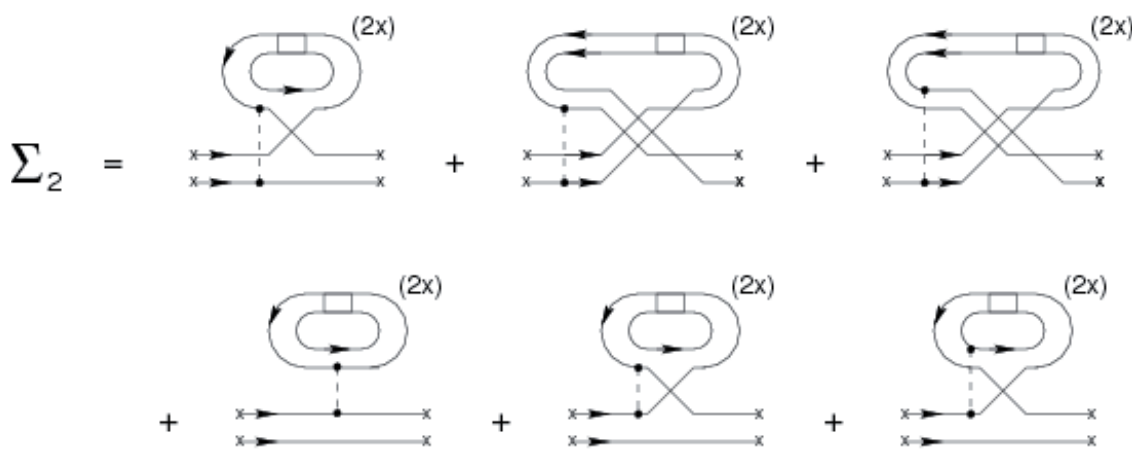

\section{Account of two-particle correlations in the medium}

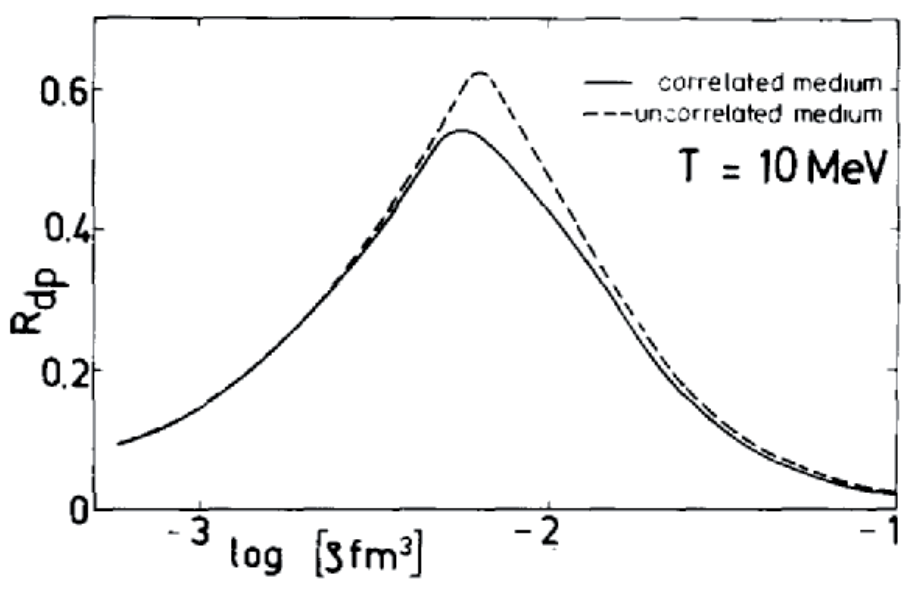




\section{Quasiparticle energy shift}

- Brueckner-Bethe-Goldstone (dashed)

- generalized Beth-Uhlenbeck (solid)

- incomplete Pauli-blocking (dotted)

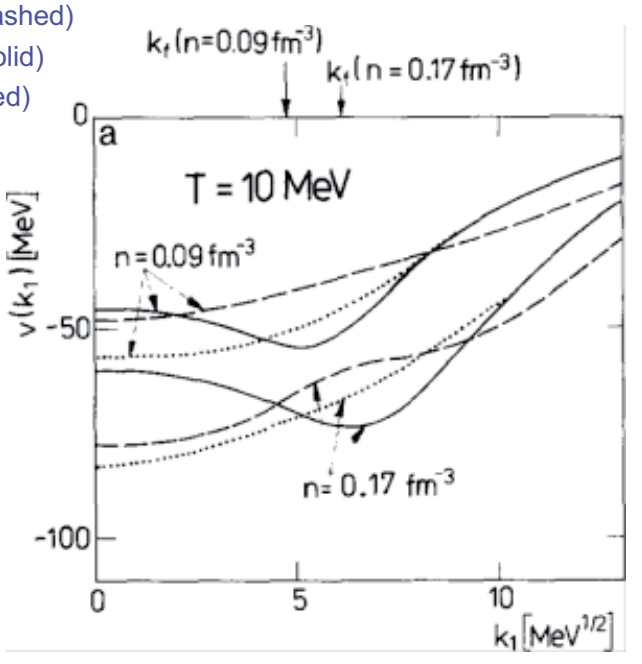

\section{Cluster decomposition of the self-energy}

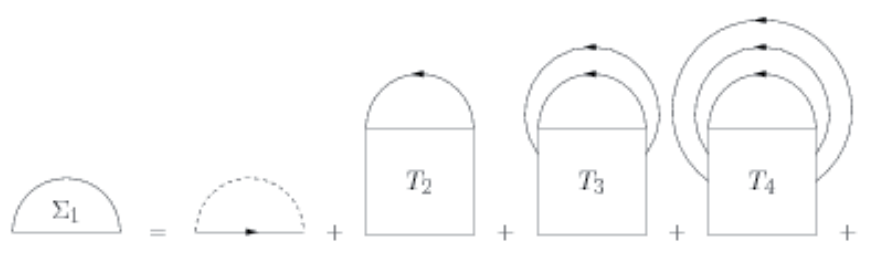




\section{Few-particle Schoedinger equation in a dense medium}

Four-particle Schrödinger equation with medium effects

$$
\begin{aligned}
& {\left[E^{\mathrm{HF}}\left(p_{1}\right)+E^{\mathrm{HF}}\left(p_{2}\right)+E^{\mathrm{HF}}\left(p_{3}\right)+E^{\mathrm{HF}}\left(p_{4}\right)\right] \psi_{n P}\left(p_{1}, p_{2}, p_{3}, p_{4}\right)} \\
& +\sum_{p_{1}^{\prime} p_{2}^{\prime} p_{3}^{\prime} p_{4}^{\prime}}\left\{\left[1-f\left(p_{1}\right)-f\left(p_{2}\right)\right] V\left(p_{1} p_{2}, p_{1}^{\prime} p_{2}^{\prime}\right) \delta_{p_{3} p_{3}^{\prime}} \delta_{p_{4} p_{4}^{\prime}}\right. \\
& \quad+\left[1-f\left(p_{1}\right)-f\left(p_{3}\right)\right] V\left(p_{1} p_{3}, p_{1}^{\prime} p_{3}^{\prime}\right) \delta_{p_{2} p_{2}^{\prime}} \delta_{p_{4} p_{4}^{\prime}} \\
& \quad+\text { permutations }\} \psi_{n P}\left(p_{1}^{\prime}, p_{2}^{\prime}, p_{3}^{\prime}, p_{4}^{\prime}\right) \\
& =E_{n P} \psi_{n P}\left(p_{1}, p_{2}, p_{3}, p_{4}\right)
\end{aligned}
$$

\section{In-medium shift of binding energies of clusters}

A. Sedrakian et al., PRC (2006), M. Beyer et al,, Phys.Lett.B 488, 247 (2000)

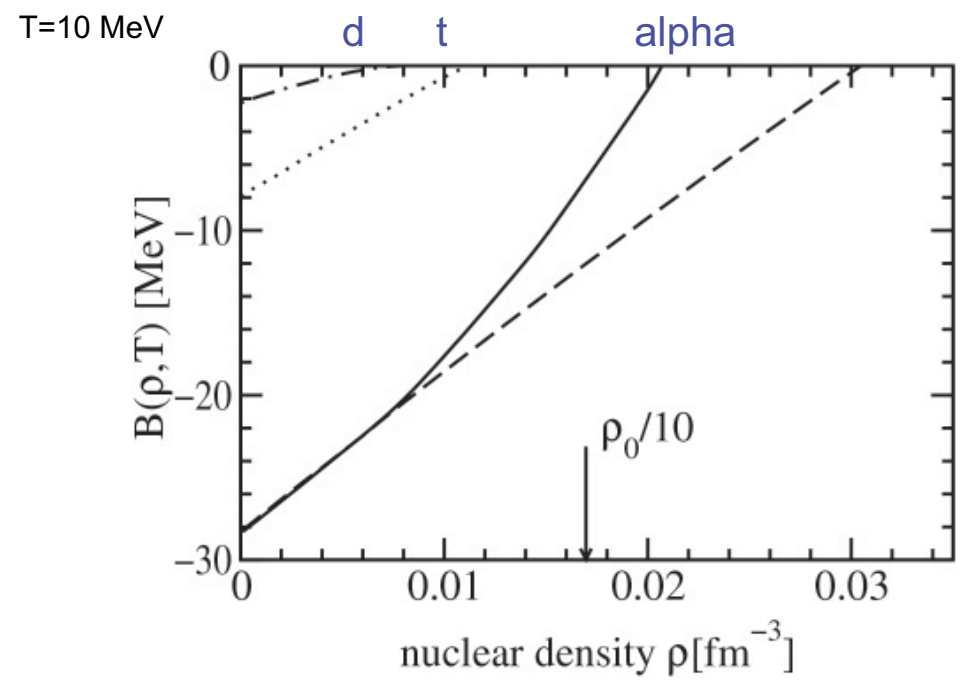


EPJ Web of Conferences

\section{Composition of symmetric nuclear matter}

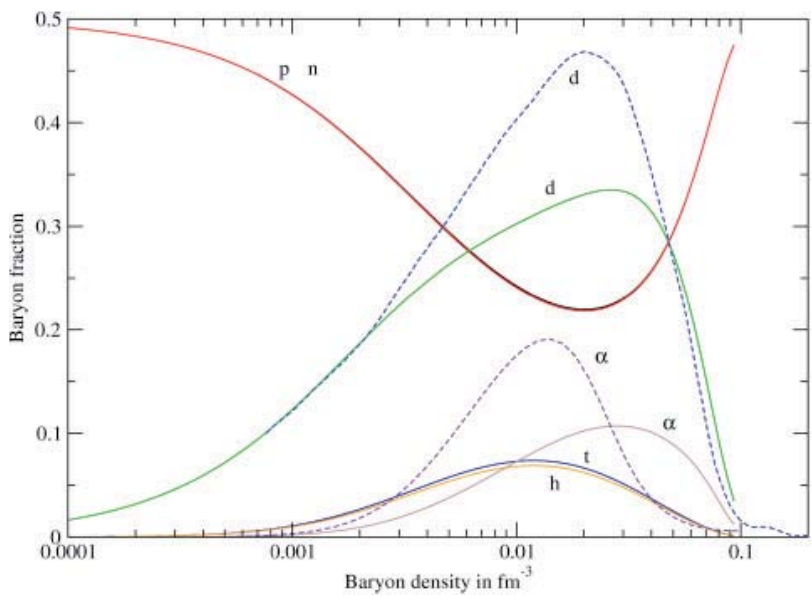

\section{Light Cluster Abundances}

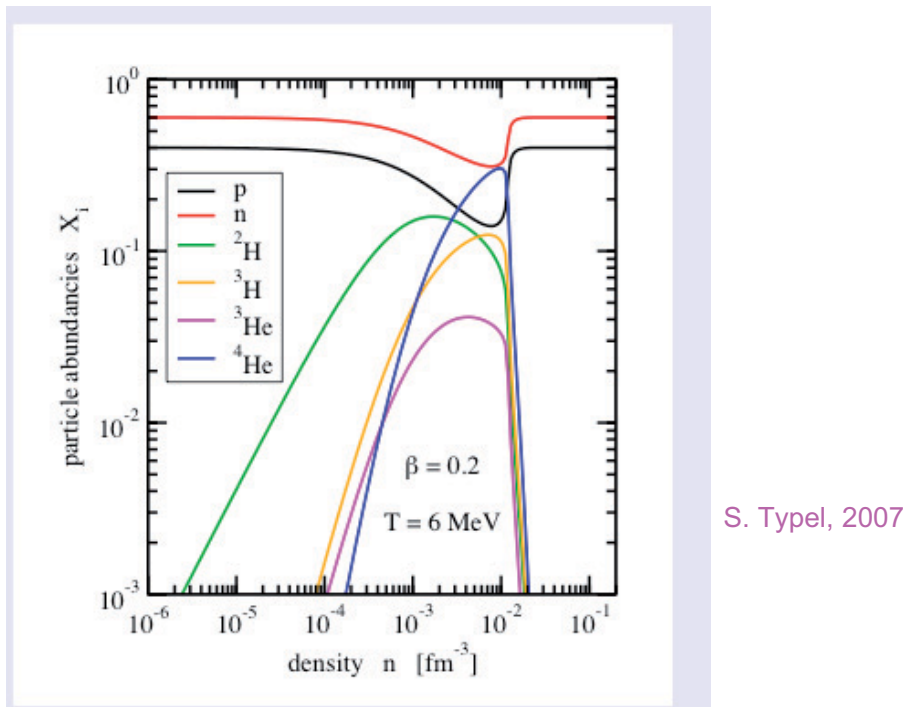




\section{Core-collapse supernovae}

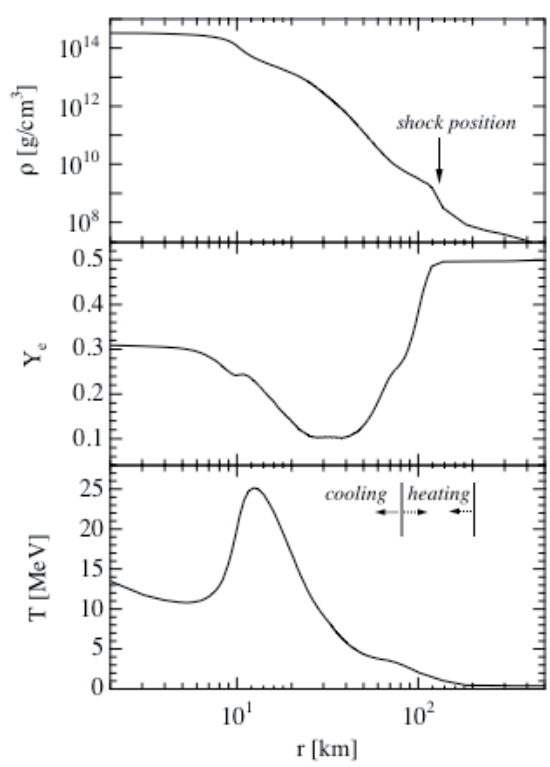

Density.

electron fraction, and

temperature profile

of a 15 solar mass supernova at $150 \mathrm{~ms}$ after core bounce as function of the radius.

Influence of cluster formation on neutrino emission in the cooling region and on neutrino absorption in the heating region?

\section{Composition of supernova core}

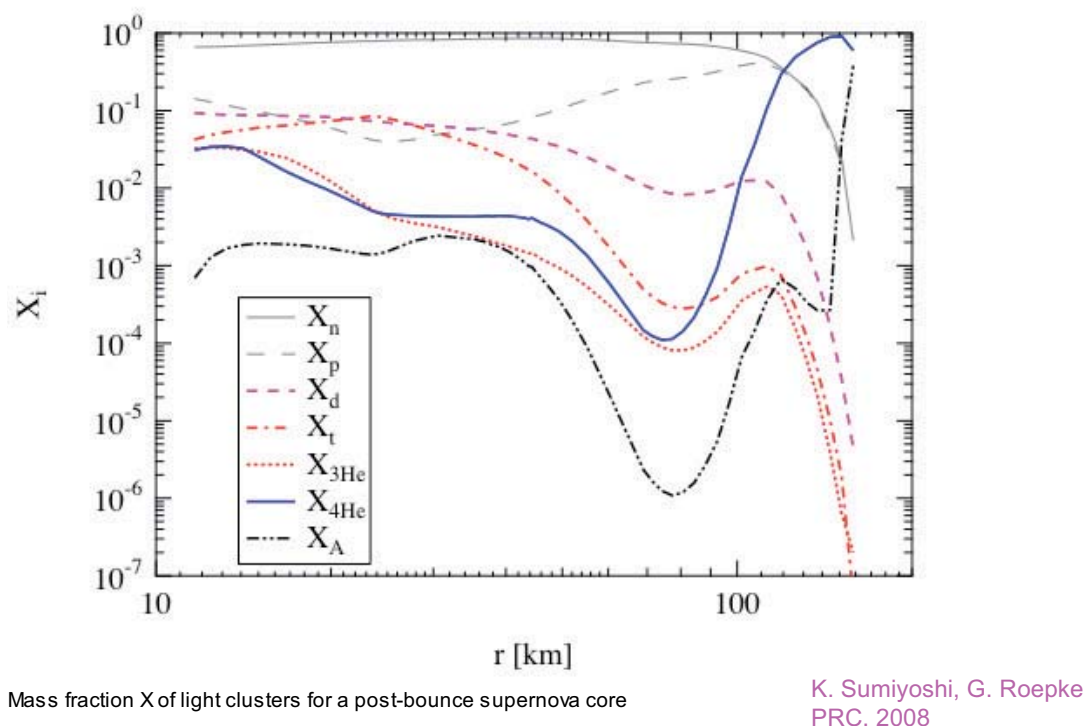


EPJ Web of Conferences

Quasiparticle approximation for nuclear matter

Equation of state for symmetric matter

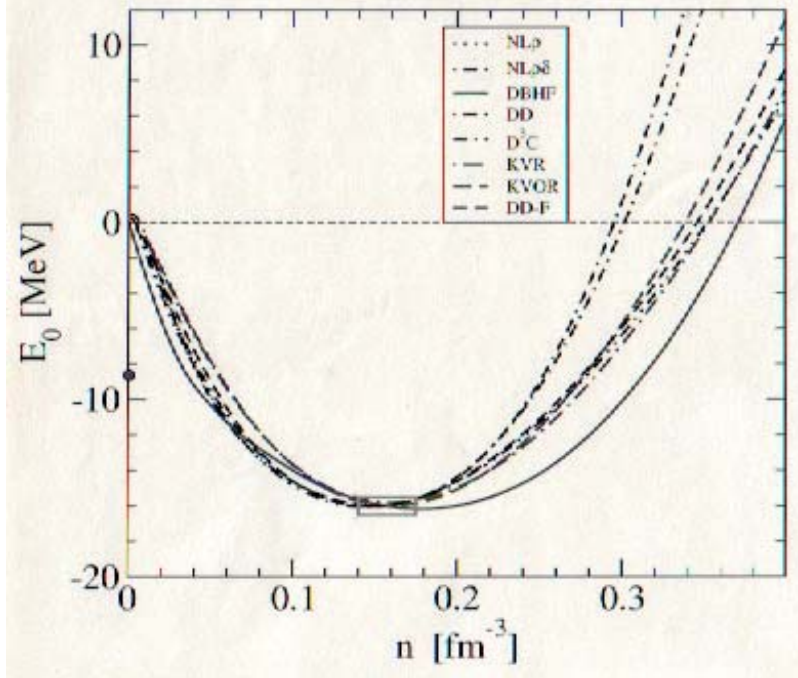

Low-density limit: alpha matter?

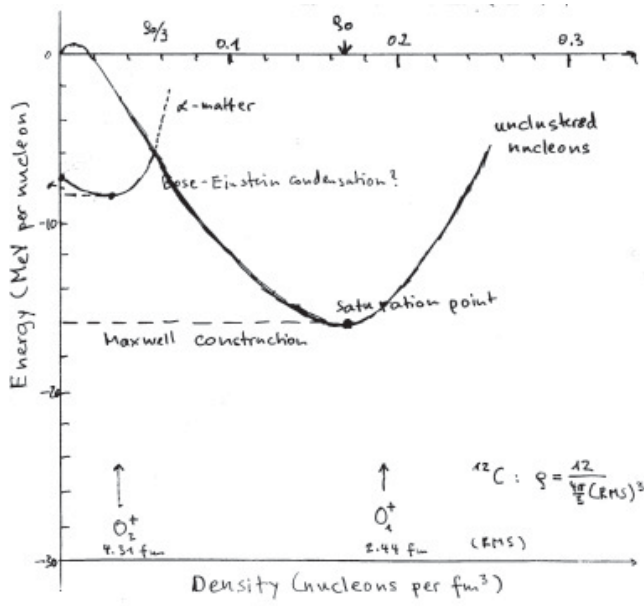




\section{Break}

- Shown today:

- correlations

- composition

- medium effects

- astrophysical relevance

- Monday's lecture:

- quantum condensates

- pairing and quartetting

- Hoyle state and low-density isomers

- low density limit of symmetry energy

HISS: Dense Matter in HIC and Astrophysics, Dubna, 18./21. 7. 08

Condensates and Correlations in Nuclear Matter

Gerd Röpke

Universität Rostock

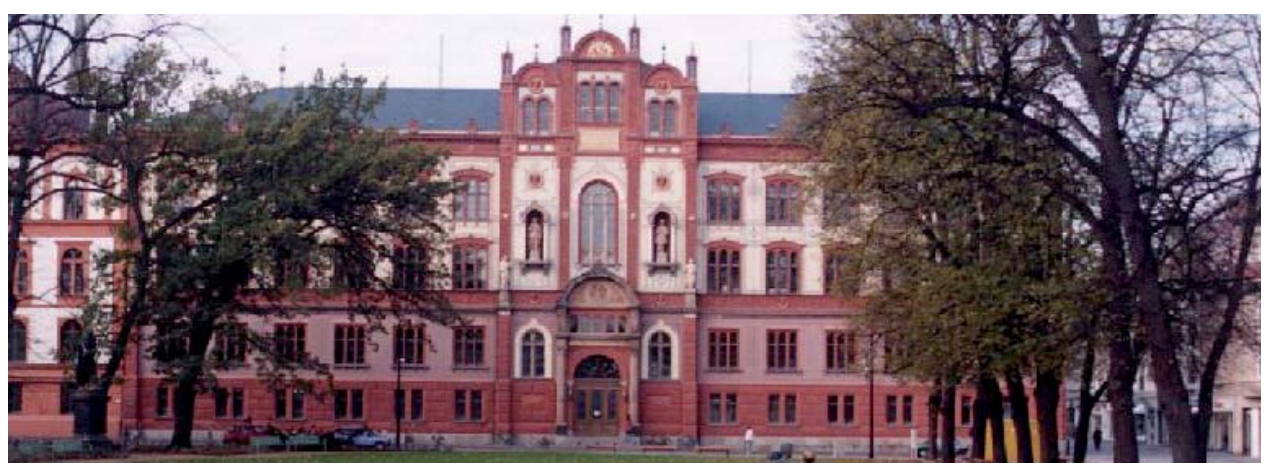




\section{Problems:}

- Warm Dilute Matter: Nuclear matter at subsaturation densities:

Temperature $T \leq 16 \mathrm{MeV}=\mathrm{E}_{\mathrm{s}} / A$, baryon density $\mathrm{n}_{\mathrm{B}} \leq 0.17 \mathrm{fm}^{-3}=\mathrm{n}_{\mathrm{s}}$.

- Formation of clusters (nuclei in matter):

$A=1,2,3,4$ : deuterons $(d)$, tritons $(t)$, helions $(h)$, alphas $(\alpha)$

- Low-density, low-temperature limit:

Virial expansion, non-interacting nuclides, quantum condensates

- Transition to higher densities:

Medium effects, quasiparticles,

interpolation between Beth-Uhlenbeck and DBHF / RMF

Refs:

Particle clustering and Mott transition in nuclear matter at finite temperatures,

G. Röpke, M. Schmidt, L. Münchow, H. Schulz: NPA 399, 587-602 (1983).

Generalized Beth-Uhlenbeck Approach for Hot Nuclear Matter,

M. Schmidt, G. Röpke, H. Schulz: Annals of Physics 202, 57 - 99 (1990).

\section{RMF and DBHF}
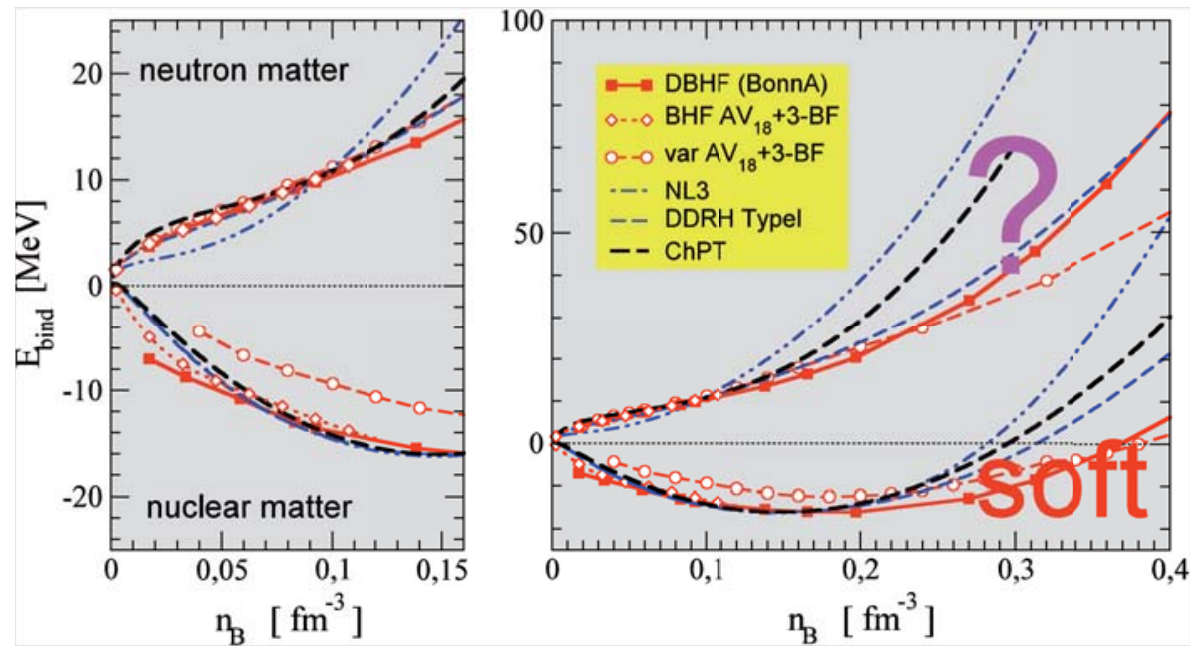

C. Fuchs et al. (2007) 
Quasiparticle approximation for nuclear matter

Equation of state for symmetric matter

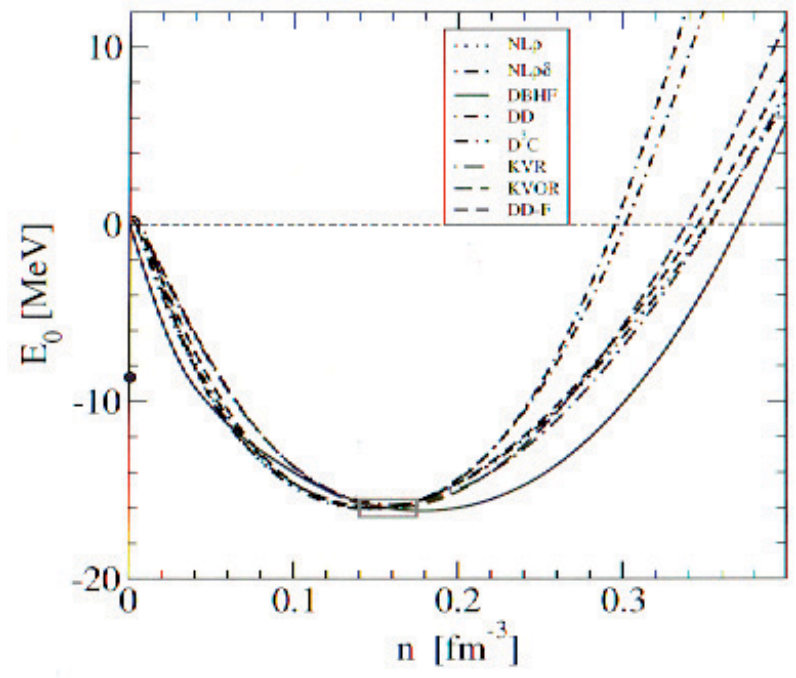

\section{Beth-Uhlenbeck formula}

rigorous results at low density: virial expansion

Beth-Uhlenbeck formula

$$
\begin{aligned}
n(T, \mu) & =\frac{1}{V} \sum_{p} e^{-(E(p)-\mu) / k_{B} T} \\
& +\frac{1}{V} \sum_{n P} e^{-\left(E_{n P}-2 \mu\right) / k_{B} T} \\
& +\frac{1}{V} \sum_{\alpha P} \int_{0}^{\infty} \frac{d E}{2 \pi} e^{-\left(E+P^{2} / 4 m-2 \mu\right) / k_{B} T} \frac{d}{d E} \delta_{\alpha}(E) \\
& +\ldots
\end{aligned}
$$

$\delta_{\alpha}(E)$ : scattering phase shifts, channel $\alpha$ 


\section{Alpha-particle fraction in the low-density limit} symmetric matter, $\mathrm{T}=4 \mathrm{MeV}$

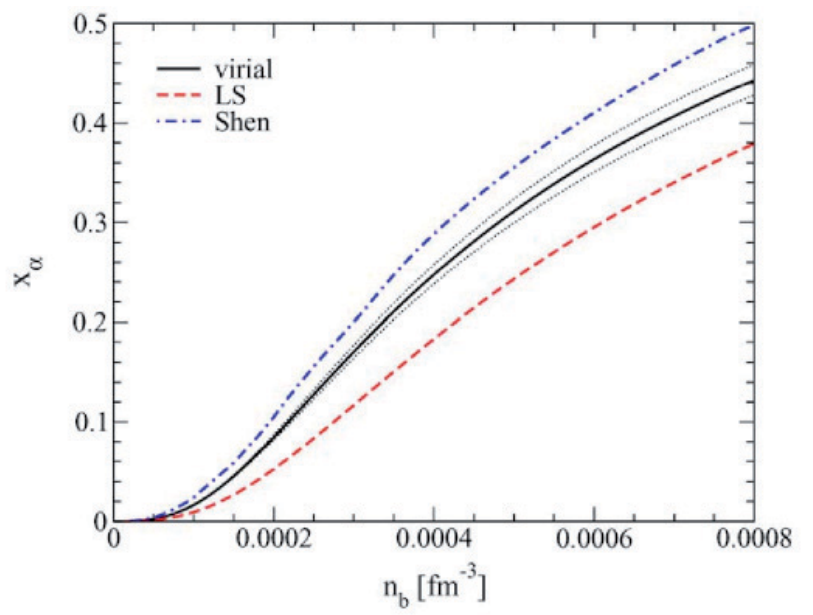

Horowitz \& Schwenk (2006), Lattimer \& Swesty, (2001),Shen et al. (1998))

Alpha-particle fraction in the low-density limit symmetric matter, $\mathrm{T}=20 \mathrm{Mev}$

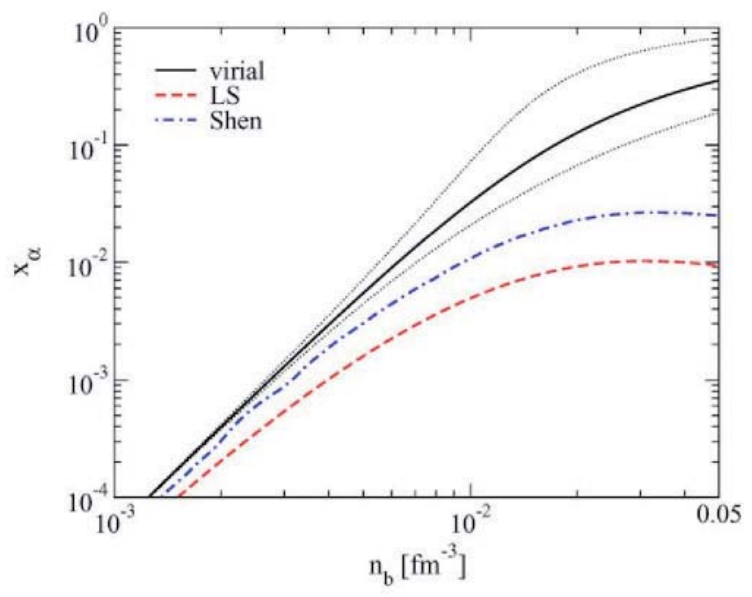




\section{Alpha-particle fraction in the low-density limit} symmetric matter, $\mathrm{T}=2,4,8 \mathrm{MeV}$

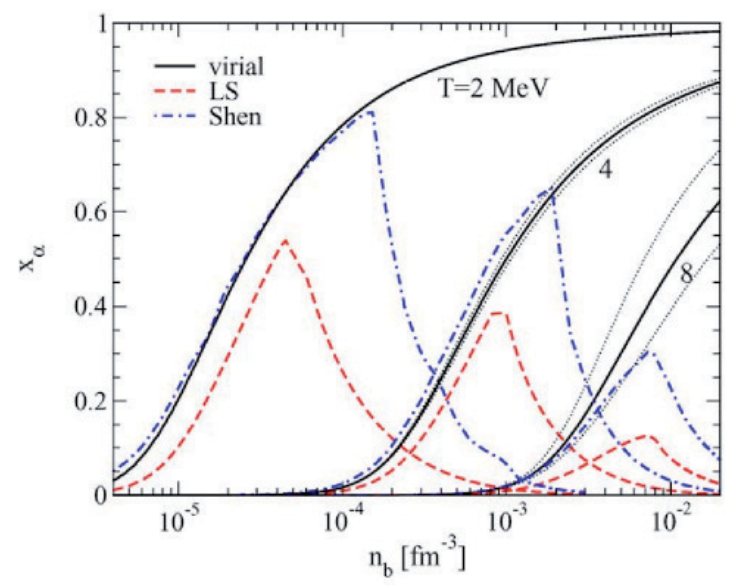

Composition of symmetric nuclear matter

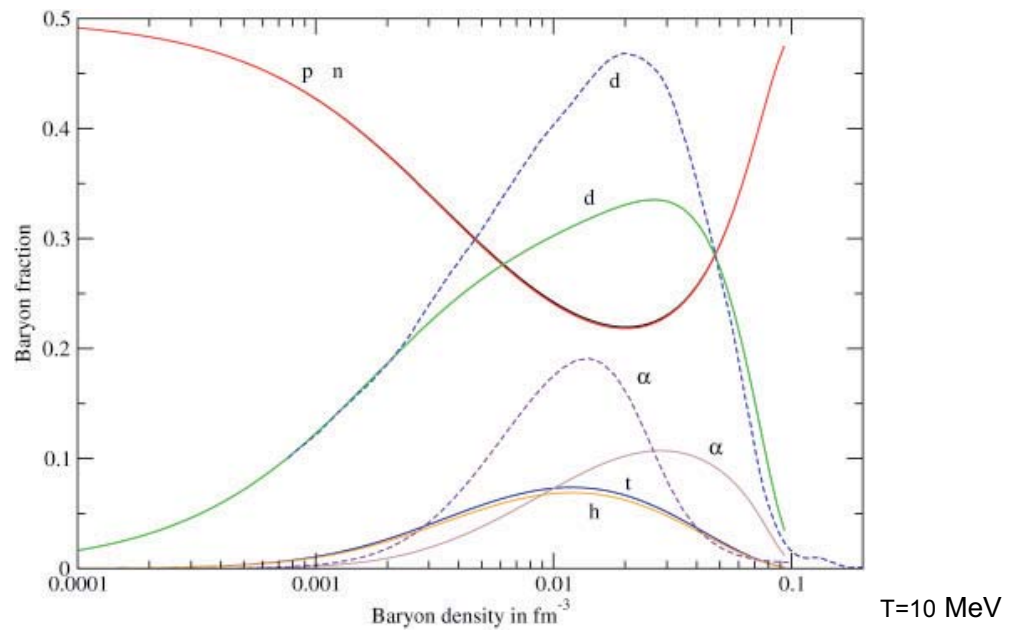




\section{$\alpha$-cluster-condensation \\ (quartetting)}

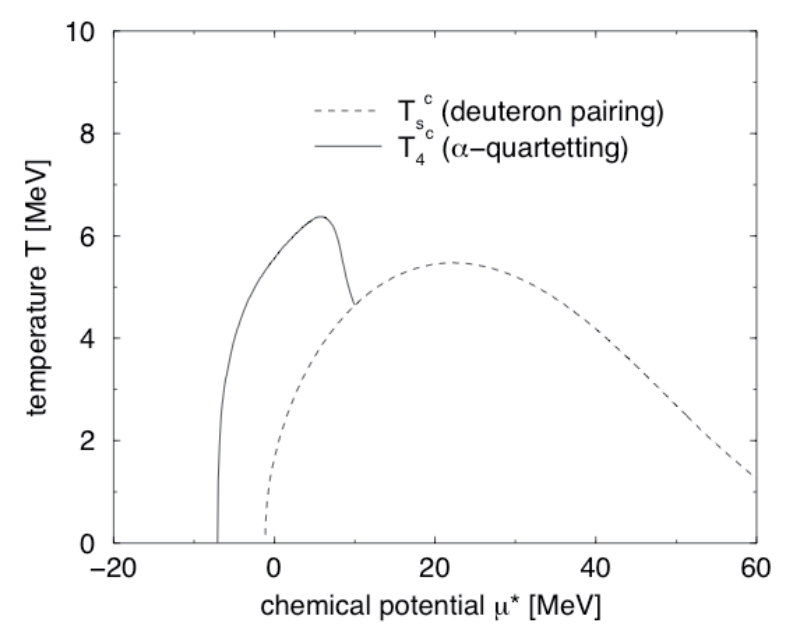

\section{$\alpha$-cluster-condensation}

(quartetting)

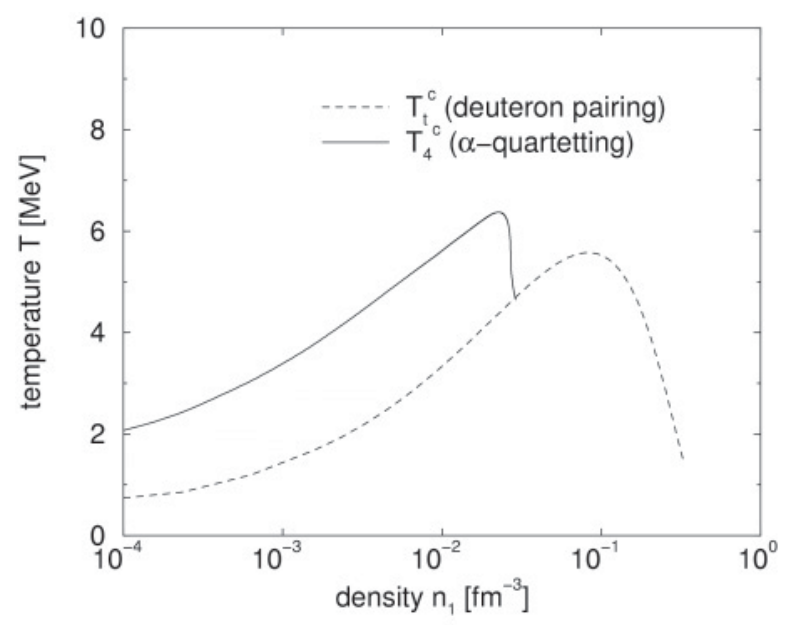




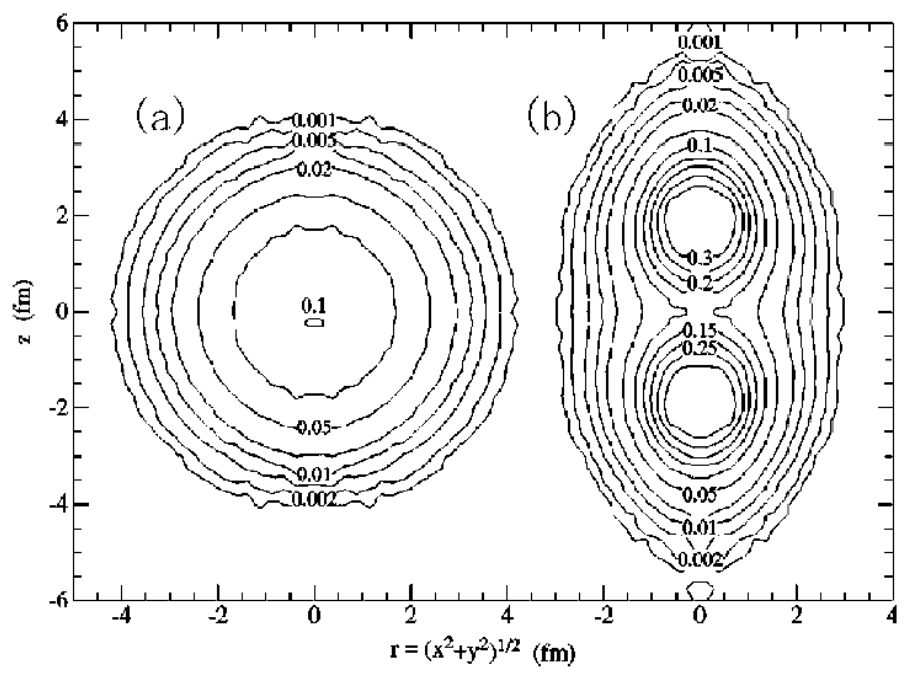

\section{Alpha-condensate (quartetting) in $4 \mathrm{n}$ symmetric nuclei}

- A. Tohsaki, H. Horiuchi, P. Schuck, G. Röpke, Phys. Rev. Lett. 87, 192501 (2001).

- G. Röpke, A. Schnell, P. Schuck, and P. Nozieres, Phys. Rev. Lett. 80, 3177 (1998).

- Y. Funaki, A. Tohsaki, H. Horiuchi, P. Schuck, G. Röpke, Phys. Rev. C 67, 051306(R) (2003); Eur. Phys. J. A 28, 259 (2006).

- T. Yamada, P. Schuck, Phys. Rev. C 69, 024309 (2004). 


\section{Self-conjugate $4 \mathrm{n}$ nuclei}

${ }^{12} \mathrm{C}$ :

$0^{+}$state at $0.39 \mathrm{MeV}$ above the $3 \alpha$ threshold energy: $\alpha$ cluster interact predominantly in relative $S$ waves, gaslike structure

$\alpha$-particle condensation in low-density nuclear matter $\left(\rho \leq \rho_{0} / 5\right)$

$n \alpha$ cluster condensed states

a general feature in $N=Z$ nuclei?

\section{Self-conjugate $4 \mathrm{n}$ nuclei}

$n \alpha$ nuclei: ${ }^{8} \mathrm{Be},{ }^{12} \mathrm{C},{ }^{16} \mathrm{O},{ }^{20} \mathrm{Ne},{ }^{24} \mathrm{Mg}, \ldots$

Single-particle shell model, or

Cluster type structures

ground state, excited states

$n \alpha$ break up at the threshold energy $E_{n \alpha}^{\mathrm{thr}}=n E_{\alpha}$ 


\section{Variational ansatz}

$$
\left|\Phi_{n \alpha}\right\rangle=\left(C_{\alpha}^{\dagger}\right)^{n}|\mathrm{vac}\rangle
$$

$\alpha$ - particle creation operator

$$
\begin{gathered}
C_{\alpha}^{\dagger}=\int d^{3} R e^{-\vec{R}^{2} / R_{0}^{2}} \\
\times \int d^{3} r_{1} \ldots d^{3} r_{4} \phi_{0 s}\left(\vec{r}_{1}-\vec{R}\right) a_{\sigma_{1} \tau_{1}}^{\dagger}\left(\vec{r}_{1}\right) \ldots \phi_{0 s}\left(\vec{r}_{4}-\vec{R}\right) a_{\sigma_{4} \tau_{4}}^{\dagger}\left(\vec{r}_{4}\right)
\end{gathered}
$$

with

$$
\phi_{0 s}(\vec{r})=\frac{1}{\left(\pi b^{2}\right)^{3 / 4}} e^{-\vec{r}^{2} /\left(2 b^{2}\right)}
$$

\section{Variational ansatz}

total $n \alpha$ wave function

$$
\begin{gathered}
\left\langle\vec{r}_{1} \sigma_{1} \tau_{1} \ldots \vec{r}_{4 n} \sigma_{4 n} \tau_{4 n} \mid \Phi_{n \alpha}\right\rangle \\
\propto \mathcal{A}\left\{e^{-\frac{2}{B^{2}}\left(\vec{X}_{1}^{2}+\ldots \vec{X}_{n}^{2}\right)} \phi\left(\alpha_{1}\right) \ldots \phi\left(\alpha_{n}\right)\right\}
\end{gathered}
$$

where $B^{2}=\left(b^{2}+2 R_{0}^{2}\right), \vec{X}_{i}=\frac{1}{4} \Sigma_{n} \vec{r}_{i n}$, $\phi\left(\alpha_{i}\right)=e^{-\frac{1}{8 b^{2}} \sum_{m>n}^{4}\left(\vec{r}_{i m}-\vec{r}_{i n}\right)^{2}}$ - internal $\alpha$ wave function

A Tohsaki, H. Horiuchi. P. Schuck. G. Röpke. PRL 87. $192501(2001)$ 


\section{3 alpha variational energy}

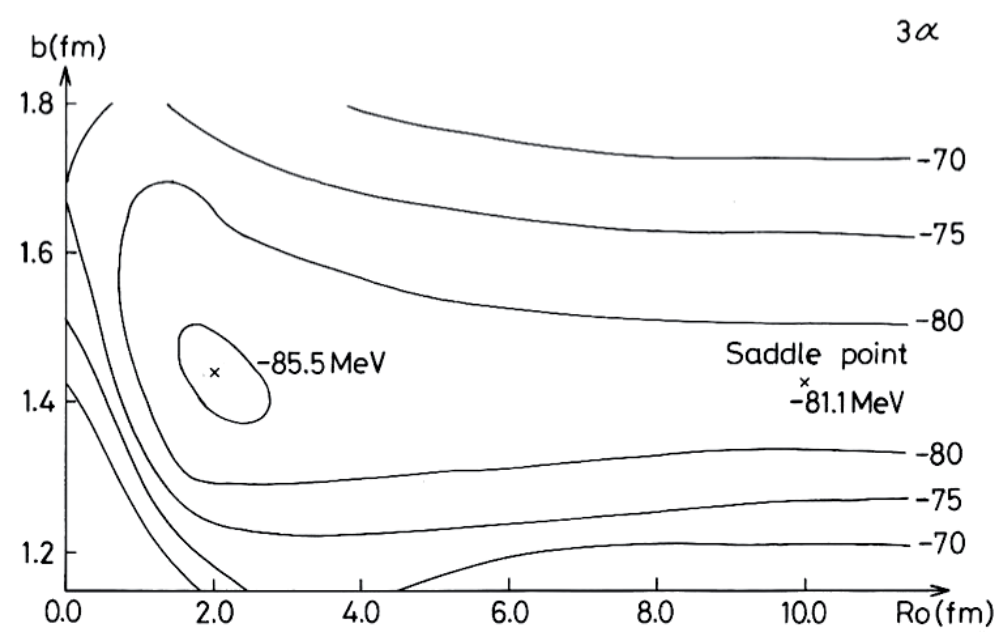

\section{4 alpha variational energy}

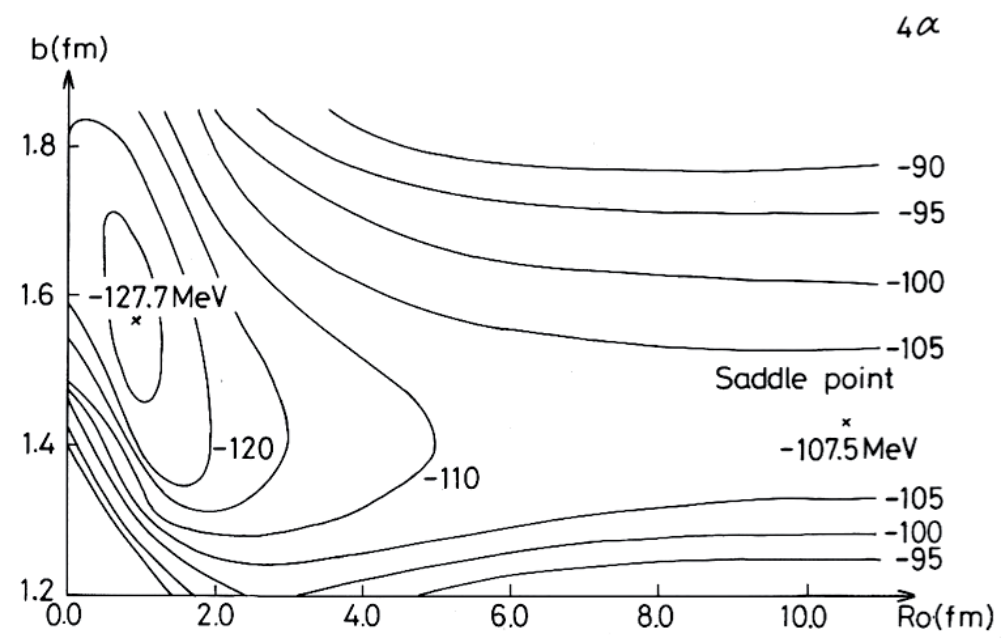




\section{Results}

\begin{tabular}{|c|c|c|c|c|c|c|}
\hline & $\begin{array}{c}E_{k} \\
(\mathrm{MeV})\end{array}$ & $\begin{array}{c}E_{\exp } \\
(\mathrm{MeV})\end{array}$ & $\begin{array}{c}E_{k}-E_{n \alpha}^{\mathrm{thr}} \\
(\mathrm{MeV})\end{array}$ & $\begin{array}{c}\left(E-E_{n \alpha}^{\text {thr }}\right)_{\exp } \\
(\mathrm{MeV})\end{array}$ & $\begin{array}{l}\sqrt{\left\langle r^{2}\right\rangle} \\
(\mathrm{fm})\end{array}$ & $\begin{array}{c}\sqrt{\left\langle r^{2}\right\rangle} \exp \\
(\mathrm{fm})\end{array}$ \\
\hline${ }^{12} \mathrm{C} \quad k=1$ & -85.9 & $-92.16\left(0_{1}^{+}\right)$ & -3.4 & -7.27 & 2.97 & 2.65 \\
\hline $\begin{array}{c}k=2 \\
E_{3 a}^{\mathrm{thr}}\end{array}$ & $\begin{array}{l}-82.0 \\
-82.5\end{array}$ & $\begin{array}{c}-84.51\left(0_{2}^{+}\right) \\
-84.89\end{array}$ & +0.5 & 0.38 & 4.29 & \\
\hline${ }^{16} \mathrm{O} \quad k=1$ & $\begin{array}{c}-124.8 \\
(-128.0)^{*}\end{array}$ & $-127.62\left(0_{1}^{+}\right)$ & $\begin{array}{c}-14.8 \\
(-18.0)^{*}\end{array}$ & -14.44 & 2.59 & 2.73 \\
\hline$k=2$ & -116.0 & $-116.36\left(0_{3}^{+}\right)$ & -6.0 & -3.18 & 3.16 & \\
\hline$k=3$ & -110.7 & $-113.62\left(0_{5}^{+}\right)$ & -0.7 & -0.44 & 3.97 & \\
\hline$E_{4 a}^{\mathrm{thr}}$ & -110.0 & -113.18 & & & & \\
\hline $\mathrm{B}_{\mathrm{BC}}$ & & & -0.17 & +0.1 & & \\
\hline
\end{tabular}

Tabelle 1: Comparison of the generator coordinate method calculations with experimental values. $E_{n \alpha}^{\text {thr }}=n E_{\alpha}$ denotes the threshold energy for the decay into $\alpha$-clusters, the values marked by ${ }^{*}$ correspond to a refined mesh.

\section{Decay of $\alpha$-condensate at high densities}

i) The bound state dissolves into free nucleon states

Occupied phase space cannot be used to form bound states

Pauli blocking $V\left(12,1^{\prime} 2^{\prime}\right)[1-f(1)-f(2)]$

$\alpha$-like bound state only below $\rho_{0} / 10$

$\alpha$-condensate survives to $\approx 0.3 \mathrm{fm}^{-3}$ 


\section{Decay of $\alpha$-condensate at high densities}

ii) Reduction of the condensate due to repulsive interaction

Penrose, Onsager 1956:

Off-diagonal long range order (ODLRO) density matrix $\rho\left(\vec{r}, \vec{r}^{\prime}\right)$ at $\left|\vec{r}-\vec{r}^{\prime}\right| \rightarrow \infty$ : $\rho\left(\vec{r}, \vec{r}^{\prime}\right)=\Psi(\vec{r}) \Psi\left(\vec{r}^{\prime}\right)+\gamma\left(\left|\vec{r}-\vec{r}^{\prime}\right|\right)$

hard core: known solution (Rosenbluth)

Application to liquid ${ }^{4} \mathrm{He}$ :

filling factor $\approx 28 \%$, condensate $\approx 8 \%$

"excluded" volume for $\alpha$ particles $\approx 20 \mathrm{fm}^{3}$

\section{Estimation of condensate fraction in zero temperature $\alpha$-matter}

$$
n_{0}=\frac{\left\langle\Psi\left|a_{0}^{\dagger} a_{0}\right| \Psi\right\rangle}{\langle\Psi \mid \Psi\rangle}
$$

destruction of the BEC of the ideal Bose gas:

thermal excitation, but also correlations

"excluded" volume for $\alpha$-particles $\approx 20 \mathrm{fm}^{3}$

at nucleon density $\rho=0.048 \mathrm{fm}^{-3}$ filling factor $\approx 28 \%$ (liquid ${ }^{4} \mathrm{He}: 8 \%$ condensate), destruction of the condensate at $\approx \rho_{0} / 3$ 


\section{Estimation of condensate fraction in zero temperature $\alpha$-matter}

$\alpha$-cluster condensate in ${ }^{12} \mathrm{C},{ }^{16} \mathrm{O}$ : resonating group method occupation numbers of $\alpha$-orbits in ${ }^{12} \mathrm{C}$

\begin{tabular}{|c|c|c|c|c|}
\hline & RMS radii & S-orbit & D-orbit & G-orbit \\
\hline $\mathrm{O}_{1}^{+}$(g.s.) & $2.44 \mathrm{fm}$ & 1.07 & 1.07 & 0.82 \\
\hline $\mathrm{O}_{2}^{+}$ & $4.31 \mathrm{fm}$ & 2.38 & 0.29 & 0.16 \\
\hline
\end{tabular}

$80 \%$ condensate at $1 / 8$ nuclear matter density

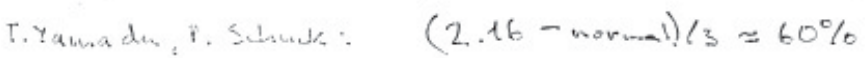

\section{Suppresion of condensate fraction}

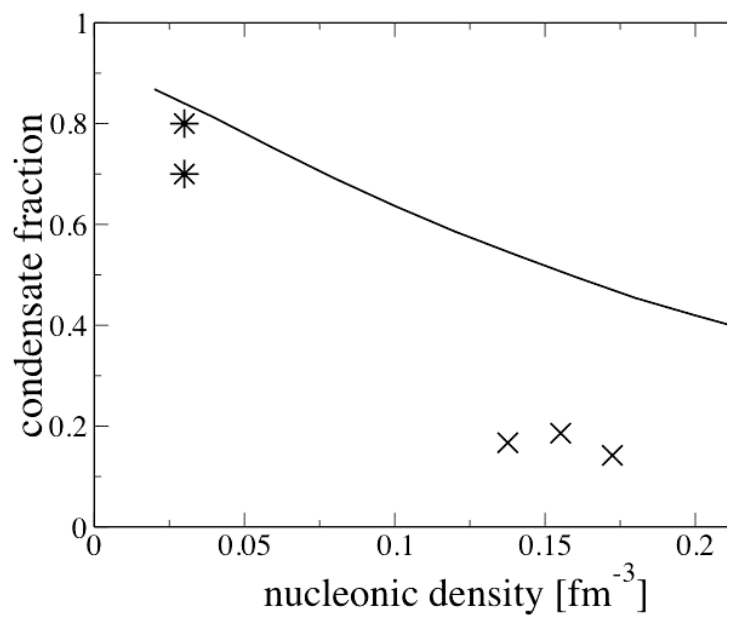


EPJ Web of Conferences

Quasiparticle approximation for nuclear matter

Equation of state for symmetric matter

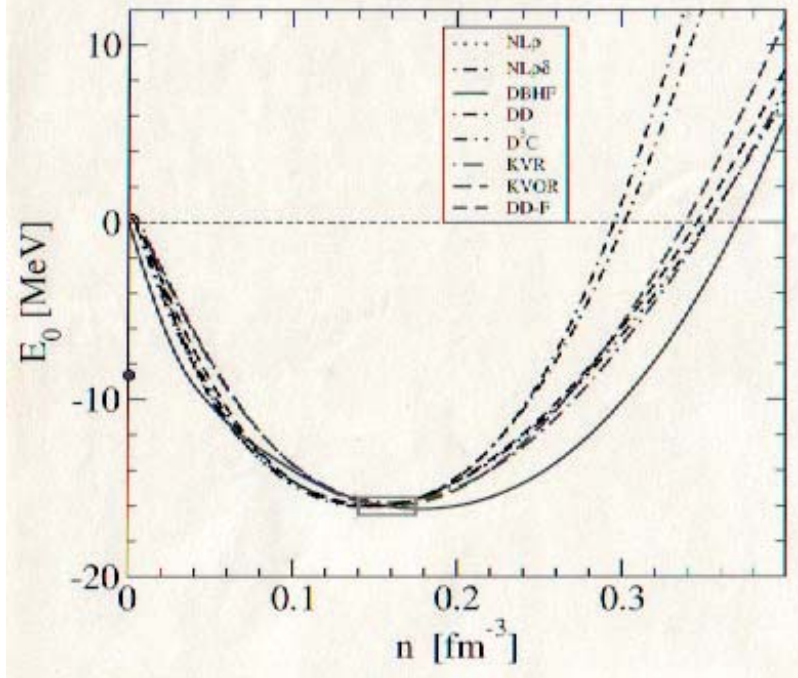

Low-density limit: alpha matter?

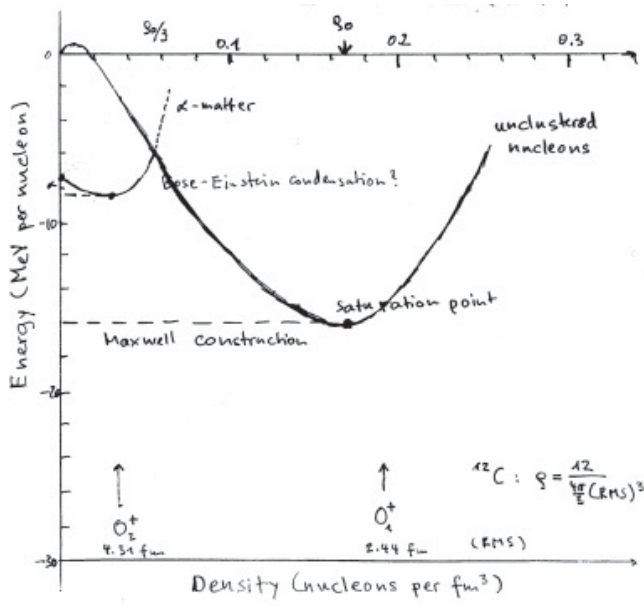




\section{Influence of cluster formation on the equation of state}

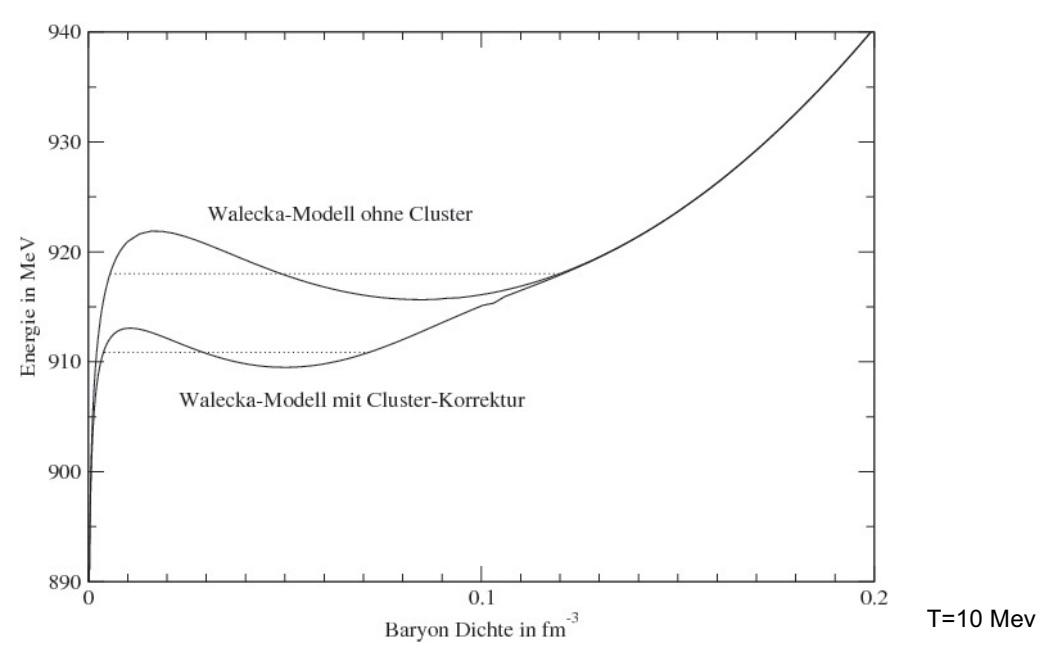

\section{Symmetry energy of a low density nuclear gas}

- L. W. Chen, C. M. Ko, and B. A. Li, Phys. Rev. Lett. 94, 032701 (2005).

- T. Klähn et al., Phys. Rev. C 74, 035802 (2006).

- C. J. Horowitz and A. Schwenk, Nucl. Phys. A 776, 55 (2006).

- S. Kowalski et al., Phys. Rev. C 75, 014601 (2007). 
Symmetry energy and single nucleon potential used in the IBUU04 transport model

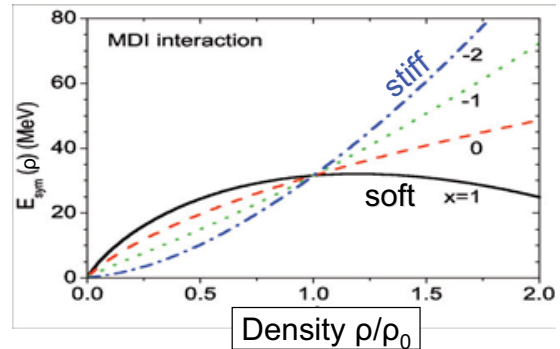

The $\mathrm{x}$ parameter is introduced to mimic various predictions by different microscopic Nuclear many-body theories using different Effective interactions

Single nucleon potential within the HF approach using a modified Gogny force:

$U(\rho, \delta, \vec{p}, \tau, x)=A_{u}(x) \frac{\rho_{\tau^{\prime}}}{\rho_{0}}+A_{l}(x) \frac{\rho_{\tau}}{\rho_{0}}+B\left(\frac{\rho}{\rho_{0}}\right)^{\sigma}\left(1-x \delta^{2}\right)-8 \tau x \frac{B}{\sigma+1} \frac{\rho^{\sigma-1}}{\rho_{0}^{\sigma}} \delta \rho_{\tau^{\prime}}$ $+\frac{2 C_{\tau, \tau}}{\rho_{0}} \int d^{3} p^{\prime} \frac{f_{\tau}\left(r, p^{\prime}\right)}{1+\left(p-p^{\prime}\right)^{2} / \Lambda^{2}}+\frac{2 C_{\tau, \tau^{\prime}}}{\rho_{0}} \int d^{3} p^{\prime} \frac{f_{\tau^{\prime}}\left(r, p^{\prime}\right)}{1+\left(p-p^{\prime}\right)^{2} / \Lambda^{2}}$

$\tau, \tau^{\prime}= \pm \frac{1}{2}, A_{l}(x)=-121+\frac{2 B x}{\sigma+1}, A_{u}(x)=-96-\frac{2 B x}{\sigma+1}, K_{0}=211 \mathrm{MeV}$

The momentum dependence of the nucleon potential is a result of the non-locality of nuclear effective interactions and the Pauli exclusion principle

C.B. Das, S. Das Gupta, C. Gale and B.A. Li, PRC 67, 034611 (2003).

B.A. Li, C.B. Das, S. Das Gupta and C. Gale, PRC 69, 034614; NPA 735, 563 (2004).

\section{$E_{\text {sym }}(\rho)$ predicted by microscopic many-body theories}

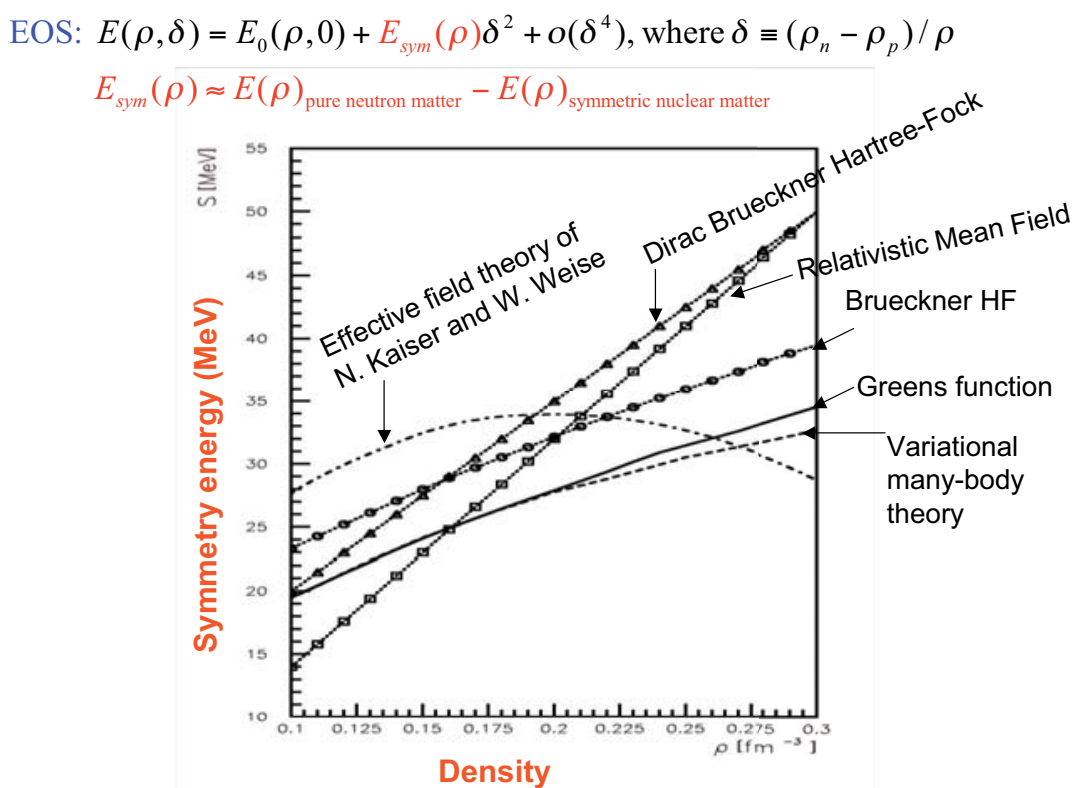

A.E. L. Dieperink, Y. Dewulf, D. Van Neck, M. Waroquier and V. Rodin, Phys. Rev. C68 (2003) 064307 
Dense Matter In Heavy Ion Collisions and Astrophysics (DM2008)

\section{Symmetry energy and symmetry free energy}

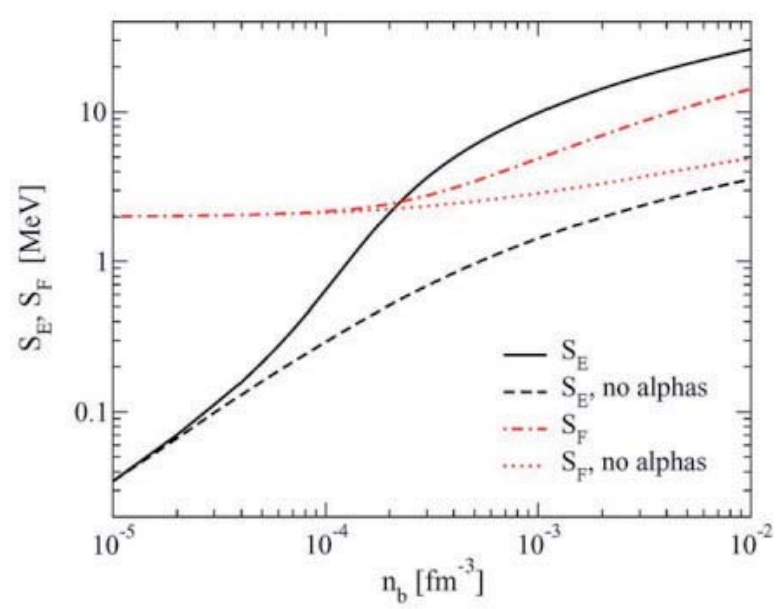

$\mathrm{T}=4 \mathrm{MeV}$

\section{Symmetry energy for $\mathrm{T}=2,4,8 \mathrm{MeV}$}

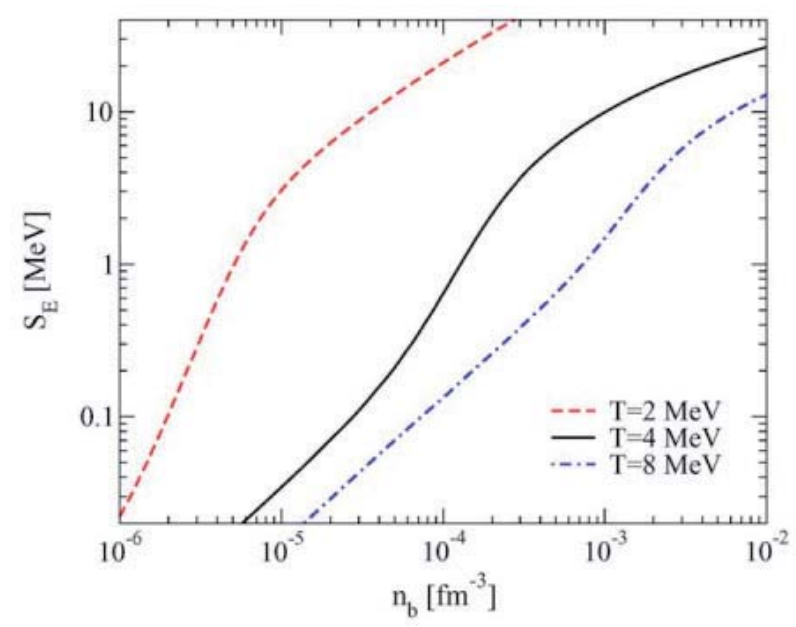


EPJ Web of Conferences

\title{
Free and Internal Symmetry Energy
}

\author{
with (solid) and without (dashed) clusters
}
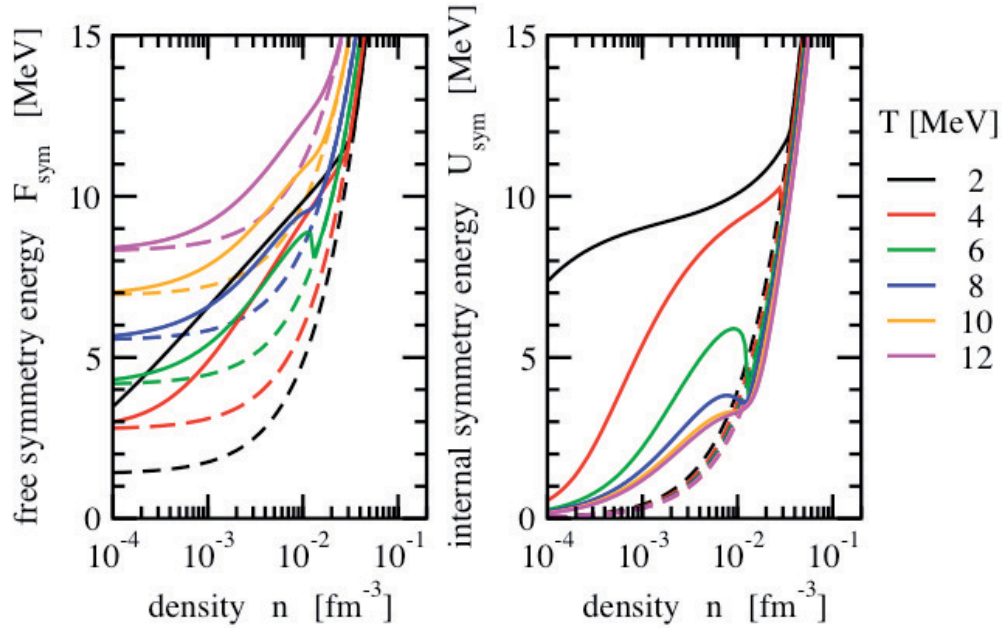

S. Typel, Talk 07

\section{Symmetry energy}

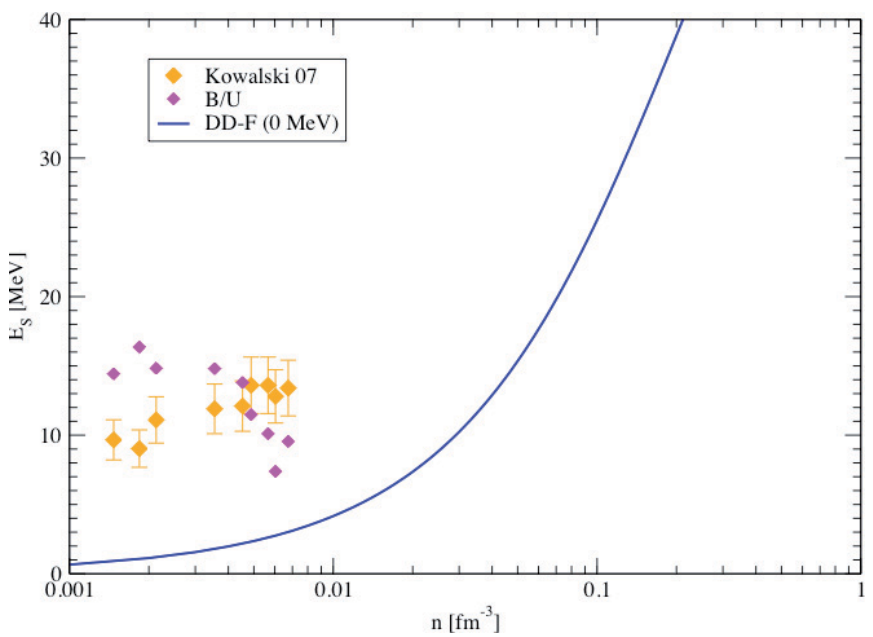




\title{
Approximations to the symmetry energy
}

\author{
a-d - Chent,Ko,Ll '0S \\ a - Kowalshi at.al 'Do
}

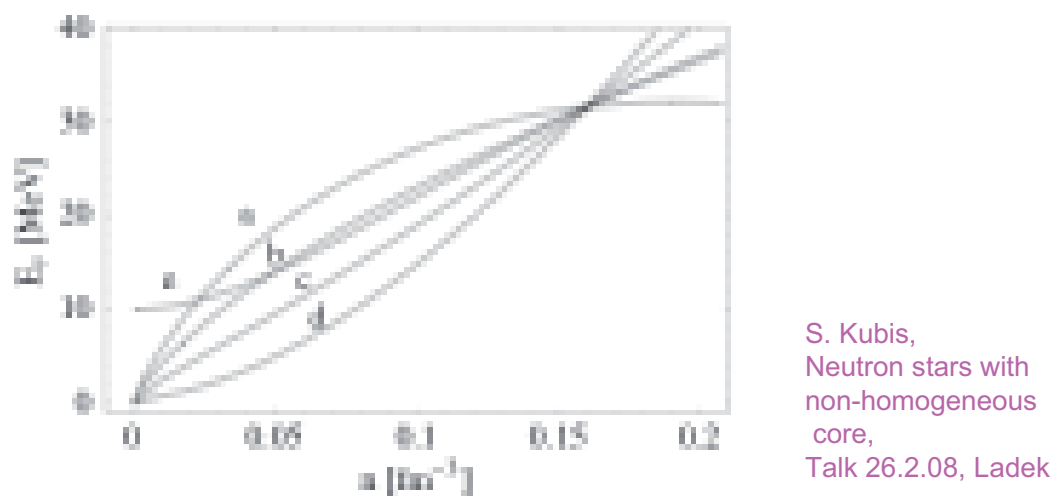

\section{Influence of cluster formation on the equation of state}

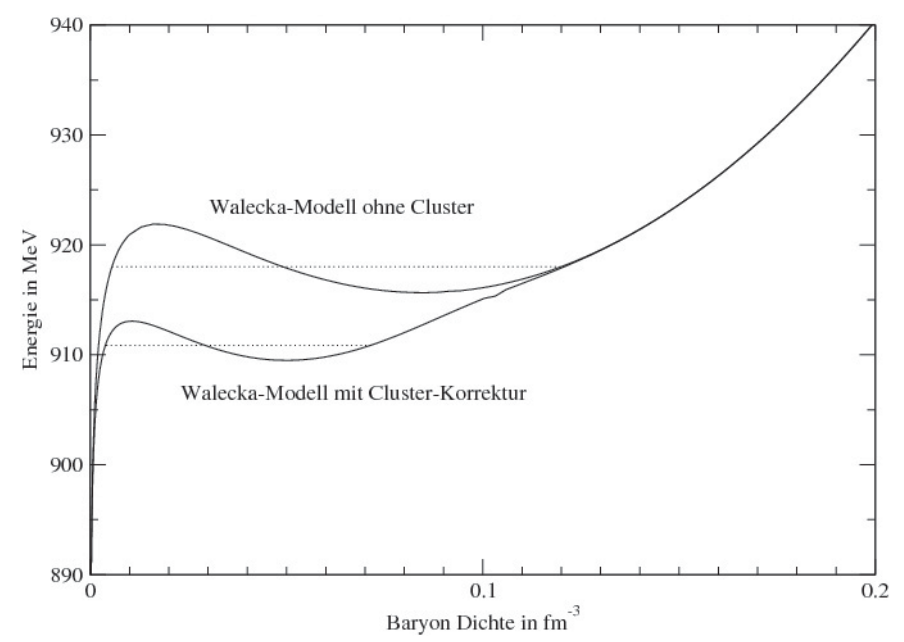

$\mathrm{T}=10 \mathrm{MeV}$

Chemical potential of symmetric nuclear matter.

Inclusion of cluster formation shifts down the chemical potential.

The region of thermodynamical instability is reduced. 


\section{Composition of symmetric nuclear matter}

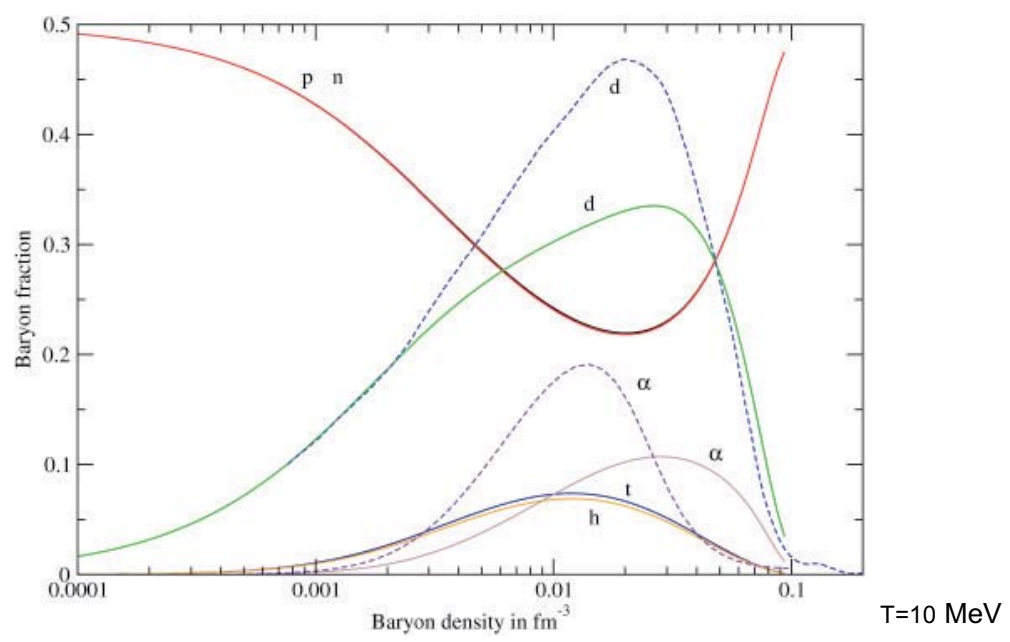

\section{Low-density EoS and astrophysics}

- H. Shen, H. Toki, K. Oyamatsu, and K. Sumiyoshi, Progr. Theor. Phys.100, 1013 (1998); Nucl. Phys. A637 435 (1998).

- G. Röpke, A. Grigo, K. Sumiyoshi, and Hong Shen, in: Superdense QCD Matter and Compact Stars, Ed. D. Blaschke and A. Sedrakian, NATO Science Series, Springer, Dordrecht (2006), pp. 75 - 91;

Physics of Particles and Nuclei Letters 2, 275 (2005).

- J.M.Lattimer and F. D. Swesty, Nucl. Phys. A 535, 331 (2001).

- C. J. Horowitz and A. Schwenk, Nucl. Phys. A 776, 55 (2006). 


\section{Outline}

- Schroedinger equation with medium corrections: Self-energy and Pauli blocking

- Composition of the nuclear gas: Generalized Beth-Uhlenbeck equation

- Quantum condensates: Pairing and quartetting

- Alpha clustering in $4 n$ nuclei

- Composition and the EoS of symmetric matter

- Symmetry energy in the low-density region

\section{Summary}

- The low-density limit of the nuclear matter EoS can be rigorously treated and has to be considered as benchmark.

- An extended quasiparticle approach can be given for single nucleon states and nuclei. In a first approximation, self- energy and Pauli blocking is included. An interpolation between low and high densities is possible.

- Compared with the standard quasiparticle approach, significant changes arise in the low-density limit due to clustering. Examples are Bose-Einstein condensation, and the behavior of the symmetry energy. 